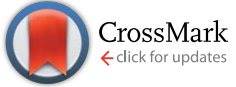

Cite this: RSC Adv., 2016, 6, 27885

Received 28th January 2016 Accepted 8th March 2016

DOI: $10.1039 / c 6 r a 02586 b$

www.rsc.org/advances

\title{
A novel acid-catalyzed rearrangement of 2- substituted-3-(2-nitrophenyl)oxiranes for the synthesis of di- and mono-oxalamides $\uparrow$
}

\author{
Vakhid A. Mamedov, ${ }^{\text {*ab }}$ Vera L. Mamedova, ${ }^{\text {ab }}$ Gul'nas Z. Khikmatova, \\ Ekaterina V. Mironova, ${ }^{a}$ Dmitry B. Krivolapov, ${ }^{a}$ Olga B. Bazanova, ${ }^{a}$ Denis V. Chachkov, ${ }^{b}$ \\ Sergey A. Katsyuba, ${ }^{a}$ Il'dar Kh Rizvanov ${ }^{a}$ and Shamil K. Latypov ${ }^{a}$ \\ A novel one-pot synthetic approach to $N^{1}$-(2-carboxyaryl)- $N^{2}$-(aryl or H)oxalamides from 3-(2-nitroaryl) \\ oxirane-2-carboxamides via the classical Meinwald rearrangement and a new rearrangement sequence \\ has been developed. The methodology is applicable to the synthesis of $\mathrm{N}$-(2-carboxyphenyl) \\ aryloxalmonoamides from (3-(2-nitrophenyl)oxiran-2-yl)(aryl)methanones. The method is operationally \\ simple and high yielding, thus providing a new useful formula for both anthranilic acid derivatives and \\ oxalamides.
}

\section{Introduction}

Oxiranes are one of the most versatile classes of organic compounds available to the synthetic chemist. ${ }^{1}$ They can be prepared by a wide variety of methods. ${ }^{2}$ One of the most frequently used atom economical reactions of oxiranes is their rearrangement to carbonyl compounds, and a number of reagents including a variety of Lewis acids ${ }^{3}$ have been elaborated for this purpose. In principle, for trisubstituted oxiranes two types of rearrangements are possible depending on the migration pathways following the Lewis acid promoted $\mathrm{C}-\mathrm{O}$ bond cleavage (Scheme 1). The rearrangement of I with hydride (path a) or the alkyl/aryl migration (path b) would lead to ketone II or aldehyde III, respectively. ${ }^{3 a, 4}$ The synthetic applications of oxiranes have been the subject of a number of reviews. ${ }^{\mathbf{a}, 2,5}$

The promise of increased chemo-, regio-, and stereoselectivity available via transition metal catalysis ${ }^{6}$ has led investigators to study the interactions of oxiranes with transition metal complexes, and a number of interesting and useful isomerization reactions have been reported. Notably, oxiranes activated by adjacent aryl, vinyl, silyl, or carbonyl substituents

${ }^{a}$ A. E. Arbuzov Institute of Organic and Physical Chemistry, Kazan Scientific Center of the Russian Academy of Sciences, Arbuzov str. 8, 420088 Kazan, Russian Federation. E-mail: mamedov@iopc.ru

${ }^{b}$ Kazan National Research Technological University, Karl Marx str. 68, 420015 Kazan, Russian Federation

$\dagger$ Electronic supplementary information (ESI) available: 1D and 2D NMR spectra and MALDI mass-spectra of the products $\mathbf{3 a}-\mathbf{h}$ and $\mathbf{4 a}-\mathbf{h}$; crystallographic data for 4a,b (CIF), description of quantum chemical computational setup and comparison of X-ray and DFT computed structural parameters of 4a. CCDC 1417946 (for 4a) and 1015263 (for 4b). For ESI and crystallographic data in CIF or other electronic format see DOI: 10.1039/c6ra02586b are isomerized to carbonyl compounds or allylic alcohols by complexes of $\mathrm{Rh},{ }^{7} \mathrm{Pd},{ }^{8} \mathrm{Mo},{ }^{9} \mathrm{Sm},{ }^{10} \mathrm{Fe}^{11}$ and $\mathrm{In} .{ }^{12}$

Unlike all the considered reactions proceeding depending on the structure of oxirane and applied conditions of rearrangement on the path a or b, our strategy included the use of oxiranes, containing substituents with functional groups instead of the usual alkyl or aryl substituents. These functional groups promote an intramolecular condensation (cyclization) of intermediate ketone II or aldehyde III formed as a result of the above two transformations. Recently, our group reported a novel metal-free transannulation reaction of $\mathrm{N}, 3$ diaryloxirane-2-carboxamides (AOCAs) involving a one-pot acidcatalyzed Meinwald rearrangement and intramolecular FriedelCrafts alkylation processes allowing to synthesize various 3-

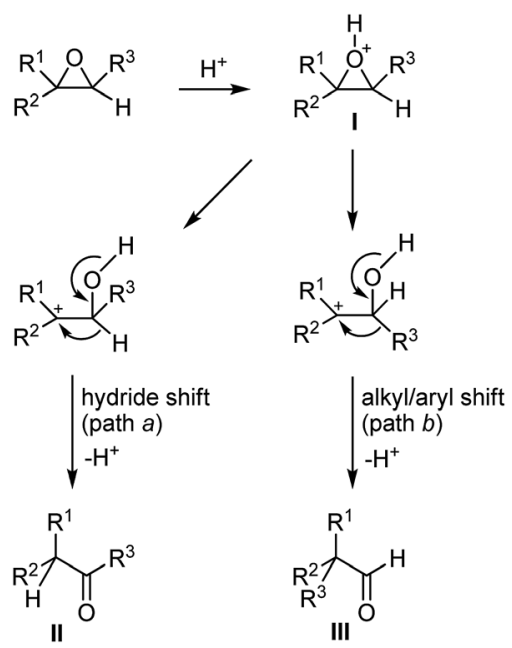

Scheme 1 Possible rearrangements of the trisubstituted oxiranes. 


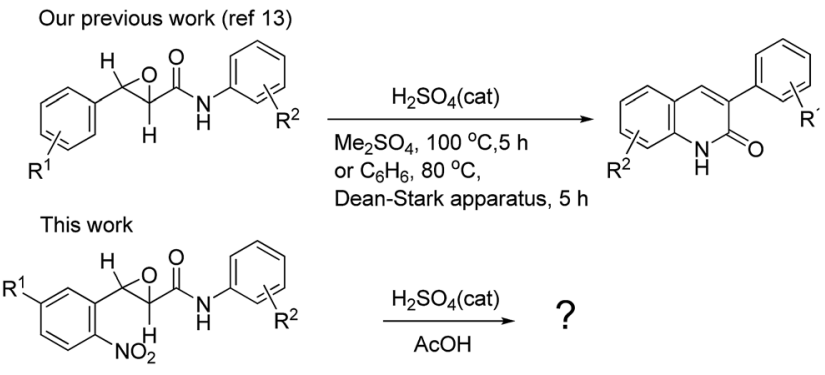

Scheme 2 Our previous work and this work.

arylquinolin-2(1H)-ones in high yields (Scheme 2). ${ }^{13}$ This novel approach features not only a metal-free bond formation but also an exclusive 1,2-aryl migration.

During our studies on the ring-opening/ring-closure reactions of AOCAs, we attempted to use $N$-aryl-3-(2-nitroaryl) oxirane-2-carboxamides (obtained from 2-nitrobenzaldehydes and 2-chloro- $N$-arylacetamides) with the aim of expanding the scope of the reaction. We found that compounds with a newly formed oxalamide chain were obtained instead of expected 3-(2nitroaryl)quinolin-2 $(1 H)$-ones when the reactions were carried out in refluxing $\mathrm{AcOH}$ in the presence of $\mathrm{H}_{2} \mathrm{SO}_{4}$. As far as we know, there has been no report on the synthesis of unsymmetrical oxalamides via the rearrangement yet. Herein, we report this novel acid-catalyzed rearrangement of AOCAs in $\mathrm{AcOH}$, which proceeds through a cascade of the ring-opening/ ring-closure/ring-closure/ring-opening/ring-opening processes.

The salient features of our method are as follows: (1) a variety of aldehydes $\mathbf{1}$ and chloroacetamides $\mathbf{2}$ are readily available and the rapid synthesis of 3 with diverse substitution patterns are possible; (2) only two steps are necessary beginning with the starting materials to the products 4 ; (3) the facile isolation of 3 and $\mathbf{4}$ are accomplished by a simple aqueous workup.

\section{Results and discussion}

The procedure of the Darzens condensation is the same as that described for AOCAs, ${ }^{13}$ except that only 2-nitrobenzaldehydes instead of variously substituted aromatic aldehydes with chloroacetamides were used and the reactions were carried out at room temperature for $7 \mathrm{~h}$. The mixtures of cis- and transisomers of 3-(2-nitroaryl)oxirane-2-carboxamides (3) with the predominance of the trans-isomer were easily purified from the cis-isomer by washing with ether (Table 1).

The structures of $3 \mathbf{a}-\mathbf{h}$ were proved by variety of $1 \mathrm{D} / 2 \mathrm{D}$ NMR correlation methods (see ESI $\dagger$ ). ${ }^{15}$ First of all to clarify the optimal reaction conditions we examined the rearrangement of trans-3-(2-nitrophenyl)- $N$-phenyloxirane-2-carboxamide (3a). After a brief survey of the reaction conditions, we have found that the product $\mathbf{4 a}$ is obtained in almost quantitative yield at reflux for $3 \mathrm{~h}$ in $\mathrm{AcOH}$ with 1 equiv. of $\mathrm{H}_{2} \mathrm{SO}_{4}$ (Table 2, entry 1). The reflux of 3a in both MeCN (with 1 equiv. $\mathrm{H}_{2} \mathrm{SO}_{4}$ ) and $\mathrm{AcOH}$ for $3 \mathrm{~h}$ resulted in the mixtures containing 30 and $10 \%$ (determined by ${ }^{1} \mathrm{H}$ NMR) of the desired product 4a, respectively. Further optimization of the reaction conditions was carried out
Table 1 Synthesis of 3-(2-nitroaryl)oxiran-2-carboxamides

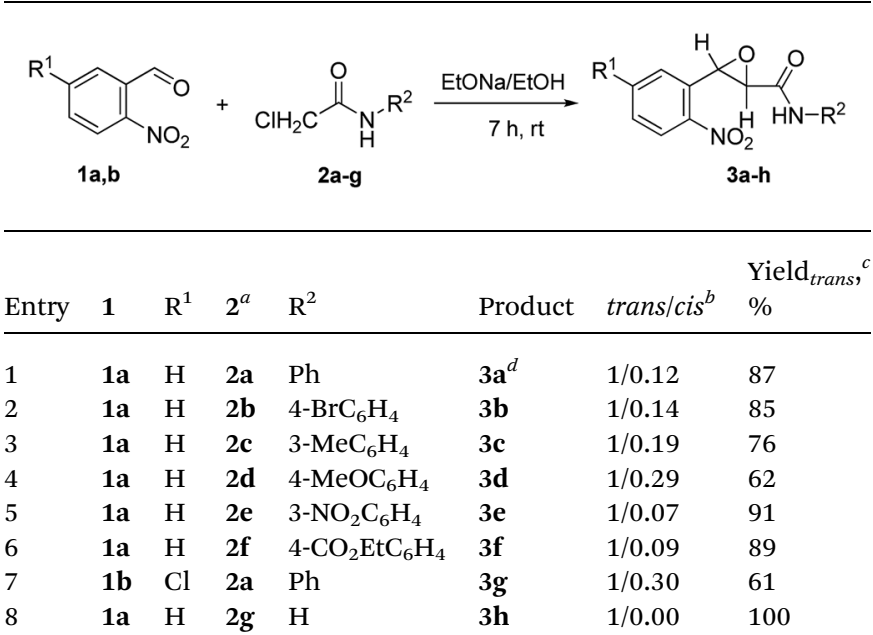

${ }^{a}$ 2-Chloro- $N$-arylacetamides $2 \mathbf{b}$-f $\mathbf{f}$ were obtained on a $0.1 \mathrm{~mol}$ scale at 0 $15{ }^{\circ} \mathrm{C}$ by reacting chloroacetylchloride with an equimolar amounts of corresponding aniline and $\mathrm{Et}_{3} \mathrm{~N}$. The compounds $2 \mathrm{a}$ and $2 \mathrm{~g}$ are commercially available. ${ }^{b}$ Ratio was determined by ${ }^{1} \mathrm{H}$ NMR of the crude products. ${ }^{c}$ Yields refer to isolated trans-isomers of $3 .{ }^{d}$ cisIsomer of this compound was obtained early. ${ }^{14}$

with trans-3-(2-nitrophenyl)oxirane-2-carboxamide (3h). The reflux of $3 \mathbf{h}$ in $\mathrm{H}_{2} \mathrm{O}$ with 1 equiv. $\mathrm{H}_{2} \mathrm{SO}_{4}$ for $5 \mathrm{~h}$ or its storage at room temp in $\mathrm{AcOH}$ with 1 equiv. of $\mathrm{H}_{2} \mathrm{SO}_{4}$ for $24 \mathrm{~h}$ gave 57 and $75 \%$ of the product $\mathbf{4 h}$, respectively. However, an almost quantitative yield of the rearrangement product was achieved when 1 equiv. of $\mathrm{H}_{2} \mathrm{SO}_{4}$ was used in boiling $\mathrm{AcOH}$ for $3 \mathrm{~h}$ (entry 8). The latter condition was used for the rearrangement of all the compounds 3 . The rearrangement proceeds equally well with the compounds 3 containing various substituents in an anilide moiety, no matter whether it is a strong electron donating (entry 4) or a strong electron withdrawing (entry 5) group. Interestingly, under the rearrangement conditions the

Table 2 Synthesis of $\mathrm{N}$-(2-carboxyaryl)oxalamides

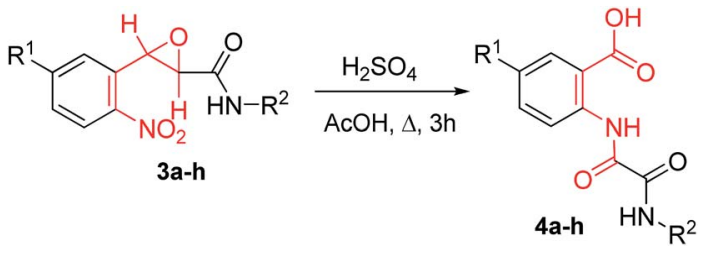

\begin{tabular}{llllll}
\hline Entry & $\mathbf{3}$ & $\mathrm{R}^{1}$ & $\mathrm{R}^{2}$ & Product & Yield, ${ }^{a} \%$ \\
\hline 1 & $\mathbf{3 a}$ & $\mathrm{H}$ & $\mathrm{Ph}$ & $\mathbf{4 a}$ & 97 \\
2 & $\mathbf{3 b}$ & $\mathrm{H}$ & $4-\mathrm{BrC}_{6} \mathrm{H}_{4}$ & $\mathbf{4 b}$ & 100 \\
3 & $\mathbf{3 c}$ & $\mathrm{H}$ & $3-\mathrm{MeC}_{6} \mathrm{H}_{4}$ & $\mathbf{4 c}$ & 92 \\
4 & $\mathbf{3 d}$ & $\mathrm{H}$ & $4-\mathrm{MeOC}_{6} \mathrm{H}_{4}$ & $\mathbf{4 d}$ & 95 \\
5 & $\mathbf{3 e}$ & $\mathrm{H}$ & $3-\mathrm{NO}_{2} \mathrm{C}_{6} \mathrm{H}_{4}$ & $\mathbf{4 e}$ & 96 \\
6 & $\mathbf{3 f}$ & $\mathrm{H}$ & $4-\mathrm{CO}_{2} \mathrm{EtC}_{6} \mathrm{H}_{4}$ & $\mathbf{4 f}$ & 97 \\
7 & $\mathbf{3 g}$ & $5-\mathrm{Cl}$ & $\mathrm{Ph}$ & $\mathbf{4 g}$ & 98 \\
8 & $\mathbf{3 h}$ & $\mathrm{H}$ & $\mathrm{H}$ & $\mathbf{4 h}$ & 97
\end{tabular}

${ }^{a}$ Yields refer to isolated products. 
ester group (entry 6) is not subjected to hydrolysis and it can be used in further transformations. The presence of the chlorine atom with the $-\mathrm{I}$ and $+\mathrm{M}$ electronic effects in the aldehyde component does not influence the yield of the rearrangement product (entry 7 ).

To our delight 3-(2-nitrophenyl)oxirane-2-carboxamides undergo the rearrangement with the formation of compounds which can be considered both as anthranilic acid and as unsymmetrical oxalamide derivatives. Anthranilic acid derivatives are potential anticancer agents ${ }^{16}$ and the ligands for farnesoid $\mathrm{X}$ receptor. ${ }^{17}$ Oxalamides also represent a key framework of many bioactive compounds. ${ }^{18}$ They have been developed as acetylcholine esterase inhibitors, ${ }^{19} \mathrm{C} 5$ a inhibitors,${ }^{20}$ nitric oxide synthase inhibitors, ${ }^{21}$ anti-HIV agents, ${ }^{22}$ antiepileptic drugs, ${ }^{23}$ HIV integrase inhibitors, ${ }^{24}$ HIV-1 proteas inhibitors, ${ }^{25}$ cephalosporin bactericides ${ }^{26}$ and chemioterapic agents. ${ }^{27}$ Considering the well documented medicinal utility of anthranilic acid and oxalamide derivatives, these tethered combinations of the two scaffolds afford new opportunities to probe their biological activity.

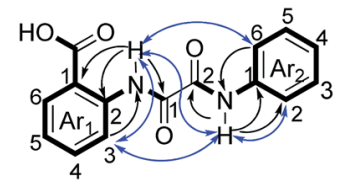

Fig. 1 Structure of $4 a$ with principle NMR correlations $\left({ }^{1} \mathrm{H}-{ }^{13} \mathrm{C} /{ }^{15} \mathrm{~N}\right.$ HMBCs - black arrow, NOEs - blue arrow).

Based on above results and literature reports, ${ }^{3 a, 4,28 a-c}$ a plausible mechanism for the rearrangement was proposed. First, the process was believed to proceed through the classical Meinwald rearrangement (Scheme 1, path $a$ ) of 3-(2-nitrophenyl)oxirane-2carboxamides with the cleavage of the $\mathrm{C} 2-\mathrm{O}$ bond in its initial stage $(3 \rightarrow \mathbf{A})$. The resulting ketone $\mathbf{A}$ bearing an active $\alpha$ methylene group undergoes a new rearrangement according to the mechanism of the known Baeyer-Drewson indigo synthe$\operatorname{sis}^{28 a-c}$ with the formation of intermediate $\mathbf{C}$, which is further subjected to acid-catalyzed ring opening (either $\mathbf{C} \rightarrow \mathbf{D} \rightarrow \mathbf{E}$ through pathway 1 or $\mathbf{C} \rightarrow \mathbf{I} \rightarrow \mathbf{J} \rightarrow \mathbf{F}$ through pathway 2)

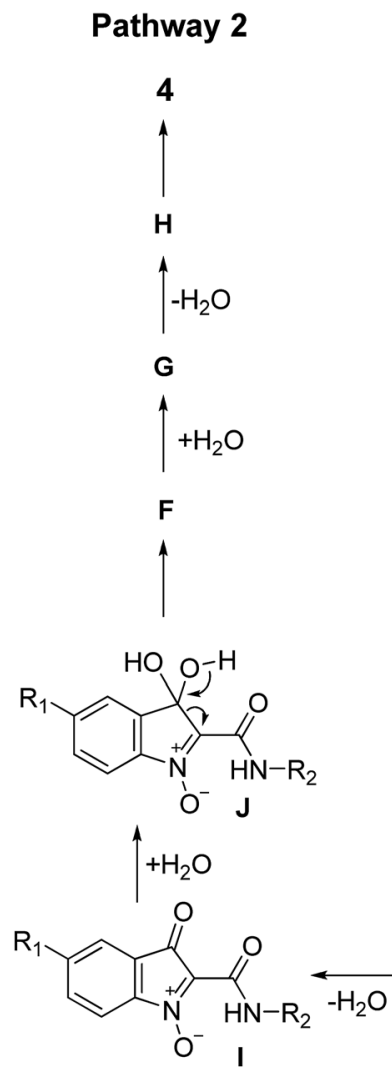<smiles>[R]NC(=O)C1(O)OC1c1cc([R])ccc1[N+](=O)[O-]</smiles><smiles>CCCCCCC</smiles><smiles>[R]NC(=O)CC(=O)c1cc([R])ccc1[N+](=O)[O-]</smiles>

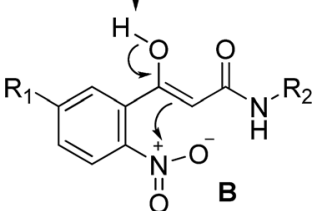<smiles>[R2]NC(=O)C1C(=O)c2cc([R])ccc2N1O[C@H](C)OC</smiles><smiles>[R]NC(=O)C(C(=O)O)(C(=O)O)N([O-])c1ccc([R])cc1C</smiles>

\section{Pathway 1}<smiles>CC(C)(C)C</smiles>

4<smiles>[R]NC(=O)c1ncccc1NC(=O)NCCO</smiles><smiles>[R]NC(=O)C(O)N(O)c1ccc([R1])cc1C(=O)O</smiles><smiles>[R]NC(=O)[C@@H](F)N([O-])c1ccc([R])cc1C(=O)O</smiles>

Scheme 3 Proposed mechanisms of the rearrangement. 


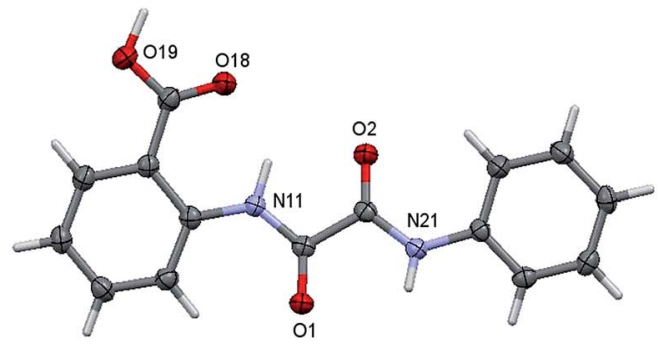

a

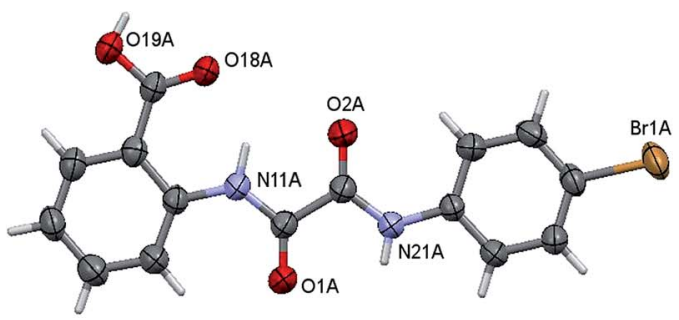

b

Fig. 2 ORTEP plot of compounds $4 \mathrm{a}(\mathrm{a})$ and $4 \mathrm{~b}$ (b) partial numbering scheme. Displacement ellipsoids are drawn at the 50\% probability level. $\mathrm{H}$ atoms are represented in stick mode for clarity.

Table 3 Synthesis of 2-(2-oxo-2-phenylacetamido)benzoic acids 7a-d

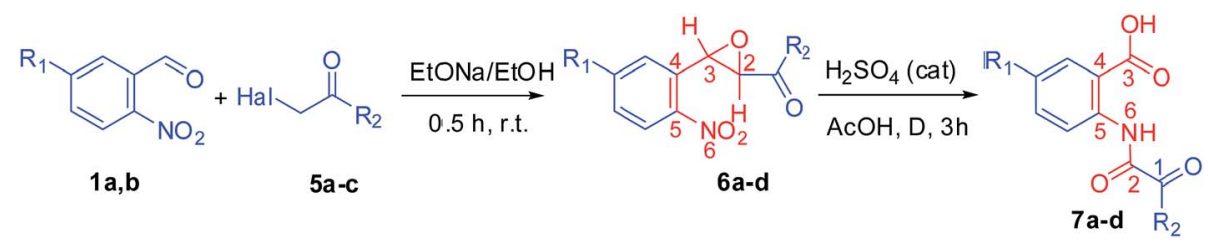

\begin{tabular}{|c|c|c|c|c|c|c|c|c|}
\hline Entry & 1 & $\mathrm{R}^{1}$ & $5^{a, b}$ & Hal & $\mathrm{R}^{2}$ & Product 6 & Product 7 & Yield, ${ }^{c} \%$ \\
\hline 1 & $1 \mathrm{a}$ & $\mathrm{H}$ & $5 a$ & $\mathrm{Cl}$ & $4-\mathrm{MeC}_{6} \mathrm{H}_{4}$ & $6 a$ & $7 \mathbf{a}$ & 75 \\
\hline 2 & $1 a$ & $\mathrm{H}$ & $5 b$ & $\mathrm{Cl}$ & $4-\mathrm{MeOC}_{6} \mathrm{H}_{4}$ & $6 b$ & $7 \mathbf{b}$ & 81 \\
\hline 4 & $1 b$ & $\mathrm{Cl}$ & $5 a$ & $\mathrm{Cl}$ & $4-\mathrm{MeC}_{6} \mathrm{H}_{4}$ & $6 d$ & $7 d$ & 89 \\
\hline
\end{tabular}

${ }^{a}$ (Chloromethyl)arylketones $5 \mathbf{a}, \mathbf{b}$ were obtained on a $0.1 \mathrm{~mol}$ scale at $10-20{ }^{\circ} \mathrm{C}$ by reaction of 1 equiv. chloroacetylchloride with toluene $(100 \mathrm{~mL})$ and anisole $\left(100 \mathrm{~mL}\right.$ ), respectively, with the use of 1.5 equiv. $\mathrm{AlCl}_{3} \cdot{ }^{b}$ (Bromomethyl)arylketone $5 \mathbf{c}$ was obtained on a $0.1 \mathrm{~mol} \mathrm{scale} \mathrm{at} 50-55{ }^{\circ} \mathrm{C}$ by reaction of 1 equiv. 1-(3-nitrophenyl)ethanone with 1 equiv. bromine in ethanol $(100 \mathrm{~mL}) .{ }^{c}$ Yields refer to isolated products.

involving hydration and dehydration processes $(\mathbf{E} \rightarrow \mathbf{F} \rightarrow \mathbf{G} \rightarrow$ $\mathbf{H} \rightarrow \mathbf{4}$ through pathway 1 and $\mathbf{F} \rightarrow \mathbf{G} \rightarrow \mathbf{H} \rightarrow \mathbf{4}$ through pathway 2) with the participation of nitro-group and $\alpha$-methylene functionalities. As a result, the reduction of the nitro-group and the transformation of the $\mathrm{C} 3$ atom of epoxide to the carboxylic functionality occurs (Scheme 3).

The structures of $\mathbf{4 a}-\mathbf{h}$ were established unambiguously by various 1D/2D NMR correlation methods. ${ }^{15}$ First, the proton spin systems of the Ar1 and Ar2 moieties were identified by COSY/TOCSY methods. After that, the structures of both halves up to carbonyl groups $\left(\mathrm{C}_{1}\right.$ and $\left.\mathrm{C}_{2}\right)$ were established (boldfaced on Fig. 1) from the ${ }^{1} \mathrm{H}-{ }^{13} \mathrm{C}$ and ${ }^{1} \mathrm{H}-{ }^{15} \mathrm{~N}$ HSQC/HMBC connectivities. Finally, both halves were linked into a single whole on the basis of the NOEs between protons of these two fragments:

Structures of the compounds $\mathbf{4 a}, \mathbf{b}$ were further confirmed by single-crystal X-ray analyses (Fig. 2).

It should be pointed out that a series of synthetic methods for oxalamides have been described in the past decades. ${ }^{29}$ However, only five examples of the synthetic methods for unsymmetrical oxalamides are known. The first is traditional and based on the condensation of corresponding carboxylic acids with amines, which needs either activating agents or conversion into more reactive derivatives. ${ }^{30}$ The next three methods include the direct amidation of isocyanates, ${ }^{31} \alpha$-keto benzotriazole $^{32}$ and trichloropyruvamides with amines. ${ }^{33}$ The fifth method is a novel one and is based on green $\mathrm{H}_{2} \mathrm{O}_{2}$ promoted oxidative amidation of 2-oxoaldehydes with amines. ${ }^{34}$ Nevertheless, these methods have several drawbacks, such as harsh conditions, expensive reagents, poor atom-efficiency and limited substrate scope. Method proposed in this study demonstrated a new, efficient and metal-free synthesis of unsymmetrical oxalamides via novel rearrangement of easily available 3-(2-nitroaryl)-oxirane-2-carboxamides.

With this result in hand, we proceeded with the study of the scope of the rearrangement. As can be seen from the suggested mechanism of the rearrangement (Scheme 3) the transformation of 3-(2-nitroaryl)oxirane-2-carboxamides to the oxalamides involves the 3-(2-nitroaryl)oxirane fragment only. Thus, seeking to expand accessible skeletal diversity using the same reaction conditions, we anticipated that use of (3-(2-nitroaryl) oxiran-2-yl)(aryl)methanones (6a-d) with the same necessary fragment would facilitate access to 2-(2-oxo-2-arylacetamido) benzoic acids (7a-d). Indeed the refluxing of oxiranes $\mathbf{6 a - d}$ in $\mathrm{AcOH}$ in the presence of catalytic amount of $\mathrm{H}_{2} \mathrm{SO}_{4}$ provides the desired mono-oxalamides $7 \mathbf{a}-\mathbf{d}$ in good yields. Moreover, the reaction of 2-nitrobenzaldehyde (1a) and 5-chloro-2- 
nitrobenzaldehyde (1) $)$ with 2-chloro-1-(4-tolyl)ethanone (5a), 2chloro-1-(4-methoxyphenyl)ethanone (5b) and 2-chloro-1-(3nitrophenyl)ethanone (5c) under the Darzens condensation condition proceeded smoothly, and (3-(2-nitrophenyl)oxiran-2yl)(aryl)methanones 6a-d were obtained in quantitative yields (Table 3). It should be pointed out that in this case, in contrast to the reactions of chloroacetanilides $2,^{13}$ the process proceeds with high stereoselectivity with the formation of only transisomers of oxiranes $\mathbf{6 a - d}$ as the only products.

The structures of all compounds were proved by variety of 1D/2D NMR correlation methods (see ESI $\dagger)^{15}$

\section{Conclusion}

In conclusion, we have discovered a new rearrangement of 3-(2nitrophenyl)-oxirane-2-carboxamides proceeding in boiling AcOH in the presence of $\mathrm{H}_{2} \mathrm{SO}_{4}$. The rearrangement quantitatively produces the $\mathrm{N}$-(2-carboxyaryl)oxalamides as a result of cascade processes involving (a) the classical Meinwald rearrangement in its initial stage with the formation of ketone bearing an active $\alpha$-methylene group, (b) transformation of carbonyl group of the ketone to the carboxylic functionality, (c) migration of active $\alpha$-methylene group to the nitrogen atom of already reduced nitro group. The simple reaction conditions offer a potential for employing this method in the synthesis of complex molecules. It is anticipated that this methodology will have versatile applications in the practical syntheses of biologically important pharmaceutical molecules with anthranilic acid and oxalamide moieties. The methodology is applicable to synthesis of $\mathrm{N}$-(2-carboxyphenyl)aryloxalmonoamides from (3(2-nitrophenyl)oxiran-2-yl)(aryl)methanones. Further extension of the reaction scope and the synthetic applications of this methodology are in progress at our laboratory.

\section{Experimental section}

\section{General methods}

All reagents and solvents were used as purchased, without further purification melting points were determined on a hotstage apparatus. Infrared (IR) spectra samples in Nujol were recorded on a FT-IR spectrometer Bruker Vector-22 in the 400$4000 \mathrm{~cm}^{-1}$ range at optical resolution of $4 \mathrm{~cm}^{-1}$. The high resolution MALDI mass-spectra were obtained on UltraFlex III TOF/TOF instrument in positive reflectron mode; 2,5-DHB and $p$-NA were used as matrix and PEG-400 was used for calibration of accurate masses. All NMR experiments were performed with 600, 500 and $400 \mathrm{MHz}$ (600 MHz for ${ }^{1} \mathrm{H}$ NMR; 150.9, 125 and 100.6 MHz for ${ }^{13} \mathrm{C} \mathrm{NMR}$; 60.8 and $50.7 \mathrm{MHz}$ for ${ }^{15} \mathrm{~N} \mathrm{NMR}$ ) spectrometers equipped with $5 \mathrm{~mm}$ diameter gradient inverse broad band probehead and a pulsed gradient unit capable of producing magnetic field pulse gradients in the $z$-direction of $53.5 \mathrm{G} \mathrm{cm}^{-1}$. NMR experiments were carried out at $303 \mathrm{~K}$. DPFGROE $^{35}$ and TOCSY spectra were obtained using a Hermiteshaped pulse for selective excitation. Chemical shifts ( $\delta$ in ppm) are referred to the solvent DMSO- $d_{6}\left(\delta=2.49 \mathrm{ppm}\right.$ for ${ }^{1} \mathrm{H}$ and $39.5 \mathrm{ppm}$ for $\left.{ }^{13} \mathrm{C} \mathrm{NMR}\right)$, to external $\mathrm{CD}_{3} \mathrm{NO}_{2}(380.2 \mathrm{ppm})$ for ${ }^{15} \mathrm{~N}$ NMR spectra (conversion factor to $\mathrm{NH}_{3}$ : $\left.-380.2 \mathrm{ppm}\right) .{ }^{1} \mathrm{H}-{ }^{1} \mathrm{H}$ coupling constants were computed according to Bally \& Rablen's recommendations. ${ }^{36}$ First the geometry was optimized at the B3LYP/6-31G(d) level. Then NMR single-point calculation of the Fermi contact $J$ values was run at the B3LYP/6-31G(d,p) level. These values were scaled then by a factor of 0.9117 . The quantum chemical calculations were performed using a Gaussian 03 software package. ${ }^{37}$

General procedure for the Darzens condensation. A solution of EtONa obtained when dissolving $\mathrm{Na}(0.73 \mathrm{~g}, 0.032 \mathrm{~g}$-atom) in EtOH $(20 \mathrm{~mL})$ was added at room temperature to the stirred solution of 2-nitrobenzaldehyde $(4.08 \mathrm{~g}, 0.027 \mathrm{~mol})$ with the corresponding compound $2(0.027 \mathrm{~mol})$ in EtOH $(70 \mathrm{~mL})$. The stirring is continued for $7 \mathrm{~h}$. Treatment of the reaction mixtures as is usual for previous cases ${ }^{\mathbf{1 3}}$ led to the products 3 .

trans-3-(2-Nitrophenyl)-N-phenyloxirane-2-carboxamides (3a).

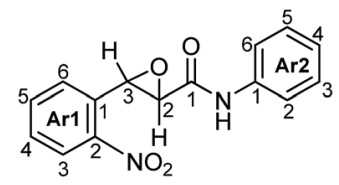

Tan powder (7.68 g, $0.027 \mathrm{~mol}, 87 \%$ yield): $\mathrm{mp} 180-181{ }^{\circ} \mathrm{C}$; ${ }^{1} \mathrm{H}$ NMR $\left(600 \mathrm{MHz}\right.$, DMSO- $\left.d_{6}\right) \delta 10.35(\mathrm{~s}, 1 \mathrm{H}, \mathrm{NH}), 8.20(\mathrm{~d}, J=8.2$ Hz, 1H, H3-Ar1), 7.85 (dd, $J=7.6,7.6$ Hz, 1H, H5-Ar1), 7.65-7.69 (m, 3H, H2,6-Ar2; H4-Ar1), 7.59 (d, $J=7.9 \mathrm{~Hz}, 1 \mathrm{H}, \mathrm{H} 6-\mathrm{Ar} 1), 7.36$ (dd, $J=7.9,7.9 \mathrm{~Hz}, 2 \mathrm{H}, \mathrm{H} 3,5-\mathrm{Ar} 2), 7.12(\mathrm{dd}, J=7.6,7.2 \mathrm{~Hz}, 1 \mathrm{H}$, H4-Ar2), 4.68 (d, $J=1.8 \mathrm{~Hz}, 1 \mathrm{H}, \mathrm{H} 3), 3.65$ (d, $J=1.8 \mathrm{~Hz}, 1 \mathrm{H}, \mathrm{H} 2)$; ${ }^{13} \mathrm{C}$ NMR (150.9 MHz, DMSO- $d_{6}$ ) $\delta 164.46$ (C1), 147.46 (C2-Ar1), 138.23 (C1-Ar2), 134.66 (C5-Ar1), 132.19 (C1-Ar1), 129.50 (C4Ar1), 128.78 (C3-Ar2), 126.73 (C6-Ar1), 124.65 (C3-Ar1), 123.91 (C4-Ar2), 119.45 (C2-Ar2), 57.37 (C2), 55.14 (C3); ${ }^{15} \mathrm{~N}$ NMR (60.8 MHz, DMSO- $\left.d_{6}\right) \delta 371.6\left(\mathrm{NO}_{2}\right), 132.3(\mathrm{NH})$; IR (nujol): $\nu 3279$, 1674, 1604, 1553, $1525 \mathrm{~cm}^{-1}$; HRMS (MALDI) calcd for $\mathrm{C}_{15} \mathrm{H}_{12} \mathrm{~N}_{2} \mathrm{O}_{4}[\mathrm{M}+\mathrm{Cs}]^{+}$416.9846, found 416.9843; anal. calcd for $\mathrm{C}_{15} \mathrm{H}_{12} \mathrm{~N}_{2} \mathrm{O}_{4}$ : C, 63.38; H, 4.25; N, 9.85; found: C, 63.42; H, 4.13; $\mathrm{N}, 9.92$.

trans-N-(4-Bromophenyl)-3-(2-nitrophenyl)oxirane-2-carboxamide (3b).

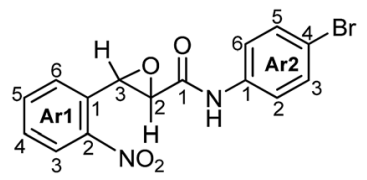

Tan powder (8.33 g, $0.023 \mathrm{~mol}, 85 \%$ yield): $\mathrm{mp} 191-192{ }^{\circ} \mathrm{C}$; ${ }^{1} \mathrm{H}$ NMR (600 MHz, DMSO- $\left.d_{6}\right): \delta 10.50(\mathrm{~s}, 1 \mathrm{H}, \mathrm{NH}), 8.19(\mathrm{dd}, J=$ 8.2, $1.0 \mathrm{~Hz}, 1 \mathrm{H}, \mathrm{H} 3-\mathrm{Ar} 1$ ), 7.85 (dd, $J=7.7,7.2 \mathrm{~Hz}, 1 \mathrm{H}, \mathrm{H} 5-\mathrm{Ar} 1$ ), 7.67 (ddd, $J=8.2,7.7,1.0 \mathrm{~Hz}, 1 \mathrm{H}, \mathrm{H} 4-\mathrm{Ar} 1), 7.64$ (d, $J=8.7 \mathrm{~Hz}$, 2H, H2,4-Ar2), 7.58 (d, $J=7.7 \mathrm{~Hz}, 1 \mathrm{H}, \mathrm{H} 6-\mathrm{Ar} 1), 7.54$ (d, $J=8.7$ $\mathrm{Hz}, 2 \mathrm{H}, \mathrm{H} 3,5-\mathrm{Ar} 2), 4.68$ (d, $J=1.8 \mathrm{~Hz}, 1 \mathrm{H}, \mathrm{H} 3$ ), 3.64 (d, $J=1.8$ $\mathrm{Hz}, 1 \mathrm{H}, \mathrm{H} 2) ;{ }^{13} \mathrm{C}$ NMR (100.6 MHz, DMSO- $\left.d_{6}\right) \delta 164.74$ (C1), 147.45 (C2-Ar1), 137.59 (C1-Ar2), 134.69 (C5-Ar1), 132.09 (C1Ar1), 131.64 (C4-Ar2), 129.56 (C4-Ar1), 126.73 (C6-Ar1), 124.68 (C3-Ar1), 121.43 (C2-Ar2), 115.61 (C4-Ar2), 57.36 (C2), 55.27 (C3); ${ }^{15} \mathrm{~N}$ NMR (60.8 MHz, DMSO- $\left.d_{6}\right) \delta 371.5\left(\mathrm{NO}_{2}\right), 131.2(\mathrm{NH}) . \mathrm{IR}$ (nujol): $\nu$ 3362, 1691, 1591, $1521 \mathrm{~cm}^{-1}$; HRMS (MALDI) calcd for $\mathrm{C}_{15} \mathrm{H}_{11} \mathrm{BrN}_{2} \mathrm{O}_{4}[\mathrm{M}+\mathrm{Cs}]^{+}$494.8951; 496.8932, found 494.8946; 
496.8938; anal. calcd for $\mathrm{C}_{15} \mathrm{H}_{11} \mathrm{BrN}_{2} \mathrm{O}_{4}$ : C, 49.61; $\mathrm{H}, 3.05 ; \mathrm{Br}$, 22.00; N, 7.71; found: C, 49.84; H, 2.99; Br, 21.84; N, 7.87.

trans-3-(2-Nitrophenyl)-N-3-tolyloxirane-2-carboxamide (3c).

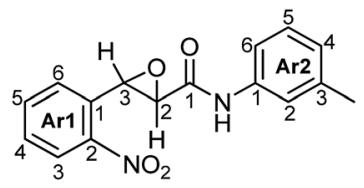

Tan powder (6.12 g, $0.021 \mathrm{~mol}, 76 \%$ yield): $\mathrm{mp} 140-143{ }^{\circ} \mathrm{C} ;{ }^{1} \mathrm{H}$ NMR (600 MHz, DMSO- $\left.d_{6}\right): \delta 10.29(\mathrm{~s}, 1 \mathrm{H}, \mathrm{NH}) ; 8.21$ (dd, $J=8.2$, $1.1 \mathrm{~Hz}, 1 \mathrm{H}, \mathrm{H} 3-\mathrm{Ar} 1$ ), 7.86 (ddd, $J=7.5,7.2,1.1 \mathrm{~Hz}, 1 \mathrm{H}, \mathrm{H} 5-\mathrm{Ar} 1$ ), 7.68 (ddd, $J=7.9,7.8,1.4 \mathrm{~Hz}, 1 \mathrm{H}, \mathrm{H} 4-\mathrm{Ar} 1), 7.59$ (d, $J=7.6 \mathrm{~Hz}, 1 \mathrm{H}$, H2-Ar1), 7.51 (br.s, 1H, H2-Ar2), 7.45 (br.d, $J=8.2 \mathrm{~Hz}, 1 \mathrm{H}, \mathrm{H6}$ Ar2), 7.24 (dd, $J=7.8,7.7 \mathrm{~Hz}, 1 \mathrm{H}, \mathrm{H} 5-\mathrm{Ar} 2), 6.94$ (d, $J=7.4 \mathrm{~Hz}$, 1H, H4-Ar2), 4.68 (d, $J=2.0 \mathrm{~Hz}, 1 \mathrm{H}, \mathrm{H} 3), 3.65$ (d, $J=2.0 \mathrm{~Hz}, 1 \mathrm{H}$, $\mathrm{H} 2), 2.31\left(\mathrm{~s}, 3 \mathrm{H}, \mathrm{CH}_{3}\right) ;{ }^{13} \mathrm{C}$ NMR $\left(125.8 \mathrm{MHz}, \mathrm{DMSO}-d_{6}\right) \delta 164.39$ (C1), 147.45 (C2-Ar1), 138.17 (C3-Ar2), 138.05 (C1-Ar2), 134.69 (C5-Ar1), 132.24 (C1-Ar1), 129.51 (C4-Ar1), 128.64 (C5-Ar2), 126.73 (C6-Ar1), 124.67 (C4-Ar2), 124.62 (C3-Ar1), 119.96 (C2-Ar2), 116.65 (C6-Ar2), 57.38 (C2), 55.14 (C3), 21.09 (Me); ${ }^{15} \mathrm{~N}$ NMR (60.8 MHz, DMSO- $\left.d_{6}\right) \delta 371.6\left(\mathrm{NO}_{2}\right), 132.5(\mathrm{NH})$; IR (nujol): $\nu$ 3252, 1667, $1611,1556,1524 \mathrm{~cm}^{-1}$; HRMS (MALDI) calcd for $\mathrm{C}_{16} \mathrm{H}_{14} \mathrm{~N}_{2} \mathrm{O}_{4}[\mathrm{M}$ $+\mathrm{Cs}]^{+}$431.0003, found 430.9994; anal. calcd for $\mathrm{C}_{16} \mathrm{H}_{14} \mathrm{~N}_{2} \mathrm{O}_{4}$ : C, 64.42; H, 4.73; N, 9.39; found: C, 64.86; H, 4.72; N, 9.41.

trans-N-(4-Methoxyphenyl)-3-(2-nitrophenyl)oxirane-2-carboxamide (3d).

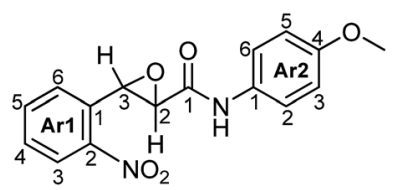

Brown powder (5.34 g, $0.017 \mathrm{~mol}, 62 \%$ yield): $\mathrm{mp} 168-169{ }^{\circ} \mathrm{C}$; ${ }^{1} \mathrm{H}$ NMR (600 MHz, DMSO- $\left.d_{6}\right): \delta 10.23(\mathrm{~s}, 1 \mathrm{H}, \mathrm{NH}), 8.21(\mathrm{dd}, J=$ 8.2, 1.1 Hz, 1H, H3-Ar1), 7.86 (ddd, $J=7.6,7.6,1.0 \mathrm{~Hz}, 1 \mathrm{H}, \mathrm{H} 5-$ Ar1), 7.68 (ddd, $J=7.8,7.8,1.3 \mathrm{~Hz}, 1 \mathrm{H}, \mathrm{H} 4-\operatorname{Ar} 1), 7.49-7.60$ (m, 3H, H2,4-Ar2; H6-Ar1), 6.93 (d, $J=9.1 \mathrm{~Hz}, 2 \mathrm{H}, \mathrm{H} 3,5-\mathrm{Ar} 2), 4.68$ (d, $J=1.9 \mathrm{~Hz}, 1 \mathrm{H}, \mathrm{H} 3), 3.75$ (s, $\left.3 \mathrm{H}, \mathrm{OCH}_{3}\right), 3.61$ (d, $J=1.9 \mathrm{~Hz}, 1 \mathrm{H}$, $\mathrm{H} 2) ;{ }^{13} \mathrm{C}$ NMR (125.8 MHz, DMSO- $d_{6}$ ) $\delta 163.98$ (C1), 155.67 (C4Ar2), 147.47 (C2-Ar1), 134.69 (C5-Ar1), 132.28 (C1-Ar1), 131.40 (C1-Ar2), 129.51 (C4-Ar1), 126.75 (C6-Ar1), 124.68 (C3-Ar1), 121.10 (C2-Ar2), 113.94 (C3-Ar2), 57.44 (C2), 55.17 (OMe), $55.10(\mathrm{C} 3) ;{ }^{15} \mathrm{~N}$ NMR $\left(60.8 \mathrm{MHz}, \mathrm{DMSO}-d_{6}\right) \delta 371.8\left(\mathrm{NO}_{2}-\mathrm{Ar} 1\right)$, 130.6 (NH); IR (nujol): $\nu$ 3268, 1664, 1608, 1556, $1514 \mathrm{~cm}^{-1}$; HRMS (MALDI) calcd for $\mathrm{C}_{16} \mathrm{H}_{14} \mathrm{~N}_{2} \mathrm{O}_{5}[\mathrm{M}+\mathrm{Na}]^{+} 337.0795$, found 337.0814; anal. calcd for $\mathrm{C}_{16} \mathrm{H}_{14} \mathrm{~N}_{2} \mathrm{O}_{5}$ : C, 61.14; H, 4.50; N, 8.91. Found: C, 61.53; H, 4.48; N, 8.93.

trans-N-(3-Nitrophenyl)-3-(2-nitrophenyl)oxirane-2-carboxamide (3e).

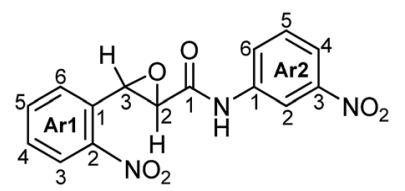

Tan powder (8.09 g, $0.025 \mathrm{~mol}, 91 \%$ yield): $\operatorname{mp~} 177{ }^{\circ} \mathrm{C} ;{ }^{1} \mathrm{H}$ NMR (600 MHz, DMSO- $\left.d_{6}\right): \delta 10.83(\mathrm{~s}, 1 \mathrm{H}, \mathrm{NH}), 8.70$ (dd, $J=2.2$, $2.2 \mathrm{~Hz}, 1 \mathrm{H}, \mathrm{H} 2-\mathrm{Ar} 2), 8.21$ (dd, $J=8.3,1.0 \mathrm{~Hz}, 1 \mathrm{H}, \mathrm{H} 3-\mathrm{Ar} 1), 8.01$ (dd, $J=8.2,1.5 \mathrm{~Hz}, 1 \mathrm{H}, \mathrm{H6}-\mathrm{Ar} 2), 7.98(\mathrm{dd}, J=8.2,1.3 \mathrm{~Hz}, 1 \mathrm{H}$, H4-Ar2), 7.86 (dd, $J=7.7,7.3 \mathrm{~Hz}, 1 \mathrm{H}, \mathrm{H} 5-\mathrm{Ar} 1$ ), 7.68 (dd, $J=7.7$, $1.4 \mathrm{~Hz}, 1 \mathrm{H}, \mathrm{H} 4-\mathrm{Ar} 1$ ), 7.66 (dd, $J=8.2,8.2 \mathrm{~Hz}, 1 \mathrm{H}, \mathrm{H} 5-\mathrm{Ar} 2), 7.60$ (d, $J=7.7 \mathrm{~Hz}, 1 \mathrm{H}, \mathrm{H} 6-\mathrm{Ar} 1), 4.73$ (d, $J=2.0 \mathrm{~Hz}, 1 \mathrm{H}, \mathrm{H} 3), 3.70$ (d, $J$ $=2.0 \mathrm{~Hz}, 1 \mathrm{H}, \mathrm{H} 2) ;{ }^{13} \mathrm{C}$ NMR $\left(150.9 \mathrm{MHz}, \mathrm{DMSO}-d_{6}\right) \delta 165.44$ (C1), 147.95 (C3-Ar2), 147.44 (C2-Ar1), 139.27 (C1-Ar2), 134.70 (C5-Ar1), 131.90 (C1-Ar1), 130.29 (C5-Ar2), 129.62 (C4-Ar1), 126.76 (C6-Ar1), 125.50 (C6-Ar2), 124.68 (C3-Ar1), 118.47 (C4Ar2), 113.72 (C2-Ar2), 57.26 (C2), 55.48 (C3); ${ }^{15} \mathrm{~N}$ NMR (60.8 MHz, DMSO- $\left.d_{6}\right) \delta 371.4\left(\mathrm{NO}_{2}-\mathrm{Ar} 1\right), 370.3\left(\mathrm{NO}_{2}-\mathrm{Ar} 2\right), 130.7(\mathrm{NH})$; IR (nujol): $\nu 3241,1673,1535,1522 \mathrm{~cm}^{-1}$; HRMS (MALDI) calcd for $\mathrm{C}_{15} \mathrm{H}_{11} \mathrm{~N}_{3} \mathrm{O}_{6}[\mathrm{M}+\mathrm{Na}]^{+}$352.0540, found 352.0559; anal. calcd for $\mathrm{C}_{15} \mathrm{H}_{11} \mathrm{~N}_{3} \mathrm{O}_{6}$ : C, 54.72; H, 3.37; N, 12.76. Found: C, 54.69; H, $3.28 ; \mathrm{N}, 12.78$.

trans-3-(2-Nitrophenyl)-N-(4-ethylcarboxyphenyl)oxirane-2-carboxamide (3f).

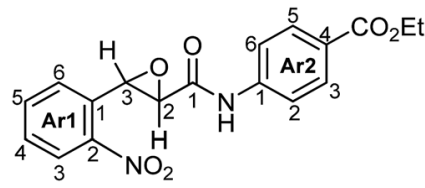

White powder (8.56 g, $0.024 \mathrm{~mol}, 89 \%$ yield): $\mathrm{mp} 180-182{ }^{\circ} \mathrm{C}$; ${ }^{1} \mathrm{H}$ NMR (600 MHz, DMSO- $d_{6}$ ) $\delta 10.86$ (br.s, $\left.1 \mathrm{H}, \mathrm{NH}\right), 8.21$ (d, $J=$ $8.2 \mathrm{~Hz}, 1 \mathrm{H}, \mathrm{H} 3-\mathrm{Ar} 1$ ), 7.96 (d, $J=8.5 \mathrm{~Hz}, 2 \mathrm{H}, \mathrm{H} 3,5-\mathrm{Ar} 2$ ), 7.86 (dd, $J$ $=7.6,7.6 \mathrm{~Hz}, 1 \mathrm{H}, \mathrm{H} 5-\mathrm{Ar} 1), 7.83$ (d, $J=8.5 \mathrm{~Hz}, 2 \mathrm{H}, \mathrm{H} 2,6-\mathrm{Ar} 2)$, 7.68 (dd, $J=8.0,7.7 \mathrm{~Hz}, 1 \mathrm{H}, \mathrm{H} 4-A r 1), 7.59$ (d, $J=7.2 \mathrm{~Hz}, 1 \mathrm{H}$, H6-Ar1), 4.71 (d, $J=1.7 \mathrm{~Hz}, 1 \mathrm{H}, \mathrm{H} 3$ ), 3.75 (d, $J=1.7 \mathrm{~Hz}, 1 \mathrm{H}, \mathrm{H} 2$ ), 4.30 (q, $J=7.1 \mathrm{~Hz}, 2 \mathrm{H}, \mathrm{OCH}_{2} \mathrm{CH}_{3}$ ), $1.33(\mathrm{t}, J=7.1 \mathrm{~Hz}, 3 \mathrm{H}$, $\left.\mathrm{OCH}_{2} \mathrm{CH}_{3}\right) ;{ }^{13} \mathrm{C}$ NMR (125.8 MHz, DMSO- $\left.d_{6}\right) \delta 165.21\left(\mathrm{CO}_{2} \mathrm{Et}\right)$, 165.17 (C1), 147.46 (C2-Ar1), 142.57 (C1-Ar2), 134.72 (C5-Ar1), 132.11 (C1-Ar1), 130.24 (C3-Ar2), 129.59 (C4-Ar1), 126.78 (C6Ar1), 124.93 (C4-Ar2), 124.70 (C3-Ar1), 118.94 (C2-Ar2), 60.46 $\left(\mathrm{OCH}_{2} \mathrm{CH}_{3}\right), 57.28$ (C2), 55.37 (C3), $14.15\left(\mathrm{OCH}_{2} \mathrm{CH}_{3}\right) ;{ }^{15} \mathrm{~N}$ NMR $\left(60.8 \mathrm{MHz}, \mathrm{DMSO}-d_{6}\right) \delta 371.7\left(\mathrm{NO}_{2}\right), 134.1(\mathrm{NH})$; IR (nujol): $\nu$ 3360, 1711, 1697, 1608, 1595, $1522 \mathrm{~cm}^{-1}$; HRMS (MALDI) calcd for $\mathrm{C}_{18} \mathrm{H}_{16} \mathrm{~N}_{2} \mathrm{O}_{6}[\mathrm{M}+\mathrm{Cs}]^{+} 489.0057$, found 489.0055; anal. calcd for $\mathrm{C}_{18} \mathrm{H}_{16} \mathrm{~N}_{2} \mathrm{O}_{6}$ : C, 60.67; H, 4.53; N, 7.86. Found: C, 60.68; $\mathrm{H}$, $4.48 ; \mathrm{N}, 7.93$.

trans-3-(2-Nitro-5-chlorophenyl)-N-phenyloxirane-2-carboxamide (3g).

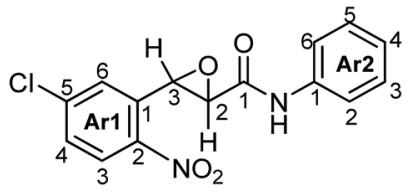

White powder $(5.25 \mathrm{~g}, 0.016 \mathrm{~mol}, 61 \%$ yield $): \operatorname{mp} 156-158{ }^{\circ} \mathrm{C}$; ${ }^{1} \mathrm{H}$ NMR (600 MHz, DMSO- $d_{6}$ ) $\delta 10.38(\mathrm{~s}, 1 \mathrm{H}, \mathrm{NH}), 8.25$ (d, $J=8.8$ $\mathrm{Hz}, 1 \mathrm{H}, \mathrm{H} 3-\mathrm{Ar} 1$ ), 7.75 (dd, $J=8.8,2.4 \mathrm{~Hz}, 1 \mathrm{H}, \mathrm{H} 4-\mathrm{Ar} 1), 7.67$ (d, $J$ $=8.4 \mathrm{~Hz}, 2 \mathrm{H}, \mathrm{H} 2,6-\mathrm{Ar} 2), 7.56(\mathrm{~d}, J=2.4 \mathrm{~Hz}, 1 \mathrm{H}, \mathrm{H} 6-\mathrm{Ar} 1), 7.38$ (dd, $J=8.4,7.5 \mathrm{~Hz}, 2 \mathrm{H}, \mathrm{H} 3,5-\mathrm{Ar} 2), 7.13$ (ddd, $J=7.5,7.4,1.0 \mathrm{~Hz}$, 1H, H4-Ar2), 4.72 (d, $J=2.0 \mathrm{~Hz}, 1 \mathrm{H}, \mathrm{H} 3), 3.72$ (d, $J=2.0 \mathrm{~Hz}, 1 \mathrm{H}$, 
$\mathrm{H} 2) ;{ }^{13} \mathrm{C}$ NMR (125.8 MHz, DMSO- $\left.d_{6}\right) \delta 164.23$ (C1), 146.12 (C2Ar1), 139.53 (C5-Ar1), 138.19 (C1-Ar2), 134.65 (C1-Ar1), 129.46 (C4-Ar1), 128.82 (C3-Ar2), 126.90 (C3-Ar1), 126.53 (C6-Ar1), 123.99 (C4-Ar2), 119.50 (C2-Ar2), 57.34 (C2), $54.84(\mathrm{C} 3) ;{ }^{15} \mathrm{~N}$ NMR (60.8 MHz, DMSO- $\left.d_{6}\right) \delta 368.9\left(\mathrm{NO}_{2}\right), 132.6$ (NH); IR (nujol): $\nu$ 3368, 1693, 1600, 1571, $1533 \mathrm{~cm}^{-1}$; HRMS (MALDI) calcd for $\mathrm{C}_{15} \mathrm{H}_{11} \mathrm{ClN}_{2} \mathrm{O}_{4}[\mathrm{M}+\mathrm{Na}]^{+}$341.0299, found 341.0308; anal. calcd for $\mathrm{C}_{15} \mathrm{H}_{11} \mathrm{ClN}_{2} \mathrm{O}_{4}$ : C, 56.53; H, 3.48; Cl, 11.12; N, 8.79. Found: C, $56.62 ; \mathrm{H}, 3.32 ; \mathrm{Cl}, 11.18 ; \mathrm{N}, 8.92$.

trans-3-(2-Nitrophenyl)oxirane-2-carboxamide (3h).

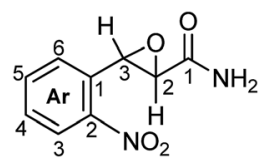

Light yellow powder (5.62 g, $0.027 \mathrm{~mol}, 100 \%$ yield): mp 209$210{ }^{\circ} \mathrm{C} ;{ }^{1} \mathrm{H}$ NMR $\left(600 \mathrm{MHz}, \mathrm{DMSO}-d_{6}\right) \delta 8.18(\mathrm{dd}, J=8.1,1.0 \mathrm{~Hz}$, 1H, H3-Ar1), 7.82 (ddd, $J=7.8,7.8,1.0 \mathrm{~Hz}, 1 \mathrm{H}, \mathrm{H} 5-\mathrm{Ar} 1$ ), 7.69 (br.s, $1 \mathrm{H}, \mathrm{NH}_{2}$ ); 7.65 (ddd, $J=8.1,7.8,1.2 \mathrm{~Hz}, 1 \mathrm{H}, \mathrm{H} 4-\mathrm{Ar} 1$ ), 7.48 (br.s, 1H, $\mathrm{NH}_{2}$ ); 7.53 (d, $\left.J=7.7 \mathrm{~Hz}, 1 \mathrm{H}, \mathrm{H6}-\mathrm{Ar} 1\right), 4.52$ (d, $J=1.9$ $\mathrm{Hz}, 1 \mathrm{H}, \mathrm{H} 3$ ), 3.39 (d, $J=1.9 \mathrm{~Hz}, 1 \mathrm{H}, \mathrm{H} 2) ;{ }^{13} \mathrm{C} \mathrm{NMR}(125.8 \mathrm{MHz}$, DMSO- $d_{6}$ ) $\delta 168.07$ (C1), 147.50 (C2-Ar), 134.68 (C5-Ar), 132.43 (C1-Ar), 129.46 (C4-Ar), 126.73 (C6-Ar), 124.68 (C3-Ar), 57.05 (C2), 54.91 (C3); ${ }^{15} \mathrm{~N}$ NMR (50.7 MHz, DMSO- $\left.d_{6}\right) \delta 371.8\left(\mathrm{NO}_{2}\right)$, $106.4\left(\mathrm{NH}_{2}\right)$; IR (nujol): $\nu$ 3368, 3185, 1665, $1526 \mathrm{~cm}^{-1}$; HRMS (MALDI) calcd for $\mathrm{C}_{9} \mathrm{H}_{8} \mathrm{~N}_{2} \mathrm{O}_{4}[\mathrm{M}+\mathrm{Cs}]^{+}$340.9533, found 340.9536; anal. calcd for $\mathrm{C}_{9} \mathrm{H}_{8} \mathrm{~N}_{2} \mathrm{O}_{4}$ : C, 51.93; $\mathrm{H}, 3.87$; N, 13.46. Found: C, 52.02; H, 3.85; N, 13.43 .

General procedure for the rearrangement. Concd $\mathrm{H}_{2} \mathrm{SO}_{4}(0.1$ $\mathrm{mL}$ ) was added to the solution of $3(1.67 \mathrm{mmol})$ in AcOH $(5 \mathrm{~mL})$. The reaction mixture was boiled for $3 \mathrm{~h}$ and then poured into water. The precipitate thus formed was collected by filtration, washed with water and dried.

$N^{1}$-(2-Carboxyphenyl)- $N^{2}$-phenyloxalamide (4a).

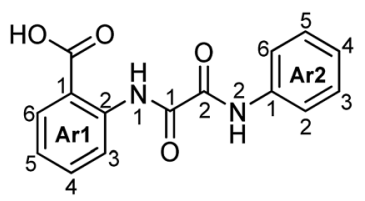

Light brown powder (0.46 g, $1.62 \mathrm{mmol}, 97 \%$ yield): $\mathrm{mp} 227-$ $228{ }^{\circ} \mathrm{C} ;{ }^{1} \mathrm{H}$ NMR $\left(600 \mathrm{MHz}, \mathrm{DMSO}-d_{6}\right) \delta 12.75$ (s, $\left.1 \mathrm{H}, \mathrm{N} 1 \mathrm{H}\right), 10.89$ (s, 1H, N2H), 8.70 (ddd, $J=8.3,8.3,1.0 \mathrm{~Hz}, 1 \mathrm{H}, \mathrm{H} 3-\mathrm{Ar} 1$ ), 8.08 (dd, $J=8.0,1.5 \mathrm{~Hz}, 1 \mathrm{H}, \mathrm{H} 6-\mathrm{Ar} 1), 7.88(\mathrm{dd}, J=8.7,1.1 \mathrm{~Hz}, 2 \mathrm{H}$, H2,6-Ar2), 7.71 (ddd, $J=7.9,7.9,1.7 \mathrm{~Hz}, 1 \mathrm{H}, \mathrm{H} 4-\mathrm{Ar} 1$ ), 7.39 (ddd, $J=8.7,7.6,1.1 \mathrm{~Hz}, 2 \mathrm{H}, \mathrm{H} 3,5-\mathrm{Ar} 2), 7.29$ (ddd, $J=7.6,7.6,1.1 \mathrm{~Hz}$, 1H, H5-Ar1), 7.18 (ddd, $J=7.4,7.4,1.0 \mathrm{~Hz}, 1 \mathrm{H}, \mathrm{H} 4-\mathrm{Ar} 2) ;{ }^{13} \mathrm{C}$ NMR (125.8 MHz, DMSO- $\left.d_{6}\right) \delta 168.84\left(\mathrm{CO}_{2} \mathrm{H}\right), 158.29(2 \mathrm{C}=\mathrm{O})$, $158.03(1 \mathrm{C}=\mathrm{O}), 139.16$ (C2-Ar1), 137.41 (C1-Ar2), 134.19 (C4Ar1), 131.43 (C6-Ar1), 128.65 (C3-Ar2), 124.72 (C4-Ar2), 123.92 (C5-Ar1), 120.60 (C2-Ar2), 119.53 (C3-Ar1), 117.39 (C1-Ar1); ${ }^{15} \mathrm{~N}$ NMR (60.8 MHz, DMSO- $d_{6}$ ) $\delta 126.8$ (N2), 121.7 (N1); IR (nujol): $\nu$ 3329, 3179, 1678, 1586, $1520 \mathrm{~cm}^{-1}$; HRMS (MALDI) calcd for $\mathrm{C}_{15} \mathrm{H}_{12} \mathrm{~N}_{2} \mathrm{O}_{4}[\mathrm{M}+\mathrm{Na}]^{+}$307.0689, found 307.0690; anal. calcd for $\mathrm{C}_{15} \mathrm{H}_{12} \mathrm{~N}_{2} \mathrm{O}_{4}$ : C, 63.38; H, 4.25; N, 9.85. Found: C, 63.34; H, 4.31; $\mathrm{N}, 9.93$.
$N^{1}$-(2-Carboxyphenyl)- $N^{2}$-(4-bromophenyl)oxalamide (4b).

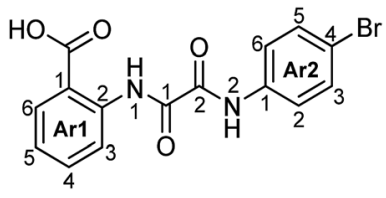

Grey powder (0.61 g, $1.67 \mathrm{mmol}, 100 \%$ yield): $\mathrm{mp} 281-283^{\circ} \mathrm{C}$; ${ }^{1} \mathrm{H}$ NMR (600 MHz, DMSO- $d_{6}$ ) $\delta 12.72(\mathrm{~s}, 1 \mathrm{H}, \mathrm{N} 1 \mathrm{H}), 11.04(\mathrm{~s}, 1 \mathrm{H}$, $\mathrm{N} 2 \mathrm{H}$ ), 8.68 (d, $J=8.2 \mathrm{~Hz}, 1 \mathrm{H}, \mathrm{H} 3-\mathrm{Ar} 1), 8.07$ (dd, $J=8.2,1.5 \mathrm{~Hz}$, 1H, H6-Ar1), 7.85 (d, $J=8.7 \mathrm{~Hz}, 2 \mathrm{H}, \mathrm{H} 2,6-\mathrm{Ar} 2$ ), 7.71 (ddd, $J=7.9$, 7.7, 1.5 Hz, 1H, H4-Ar1), 7.57 (d, $J=8.7 \mathrm{~Hz}, 2 \mathrm{H}, \mathrm{H} 3,5-\mathrm{Ar} 2), 7.28$ (ddd, $J=7.7,7.7,1.0 \mathrm{~Hz}, 1 \mathrm{H}, \mathrm{H} 5-\mathrm{Ar} 1) ;{ }^{13} \mathrm{C}$ NMR $(150.9 \mathrm{MHz}$, DMSO- $\left.d_{6}\right) \delta 168.82\left(\mathrm{CO}_{2} \mathrm{H}\right), 158.18(2 \mathrm{C}=\mathrm{O}), 158.03(1 \mathrm{C}=\mathrm{O})$, 139.10 (C2-Ar1), 136.84 (C1-Ar2), 134.20 (C4-Ar1), 131.49 (C3Ar2), 131.41 (C6-Ar1), 123.95 (C5-Ar1), 122.54 (C2-Ar2), 119.54 (C3-Ar1), 117.32 (C1-Ar1), 116.65 (C4-Ar2); ${ }^{15} \mathrm{~N}$ NMR (60.8 MHz, DMSO- $d_{6}$ ) $\delta 126.6(\mathrm{~N} 2), 122.2$ (N1); IR (nujol): $\nu 3301,3189,1687$, 1584, $1519 \mathrm{~cm}^{-1}$; HRMS (MALDI) calcd for $\mathrm{C}_{15} \mathrm{H}_{11} \mathrm{BrN}_{2} \mathrm{O}_{4}[\mathrm{M}+$ $\mathrm{Na}]^{+} 384.9794$; 386.9776, found 384.9795; 386.9786; anal. calcd for $\mathrm{C}_{15} \mathrm{H}_{11} \mathrm{BrN}_{2} \mathrm{O}_{4}$ : C, 49.61; H, 3.05; Br, 22.00; N, 7.71. Found: $\mathrm{C}$, 49.38; H, 3.02; Br, 22.07; N, 7.69.

$N^{1}$-(2-Carboxyphenyl)- $N^{2}$-(3-methylphenyl)oxalamide (4c).

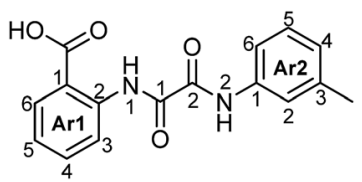

Brown powder (0.46 g, $1.54 \mathrm{mmol}, 92 \%$ yield): $\mathrm{mp} 203-205{ }^{\circ} \mathrm{C}$; ${ }^{1} \mathrm{H}$ NMR (600 MHz, DMSO- $d_{6}$ ) $\delta 12.72(\mathrm{~s}, 1 \mathrm{H}, \mathrm{N} 1 \mathrm{H}), 10.79(\mathrm{~s}, 1 \mathrm{H}$, $\mathrm{N} 2 \mathrm{H}), 8.70$ (d, $J=8.4,1.0 \mathrm{~Hz}, 1 \mathrm{H}, \mathrm{H} 3-\mathrm{Ar} 1), 8.08$ (dd, $J=7.9,1.6$ Hz, 1H, H6-Ar1), 7.73 (br.s, 1H, H2-Ar2), 7.72 (ddd, $J=7.8,7.8$, $1.7 \mathrm{~Hz}, 1 \mathrm{H}, \mathrm{H} 4-\mathrm{Ar} 1$ ), 7.64 (br.d, $J=8.3 \mathrm{~Hz}, 1 \mathrm{H}, \mathrm{H6}-\mathrm{Ar} 1$ ), 7.29 (ddd, $J=7.9,7.9,1.2 \mathrm{~Hz}, 1 \mathrm{H}, \mathrm{H} 5-\mathrm{Ar} 1$ ), 7.27 (dd, $J=7.8,7.8 \mathrm{~Hz}, 1 \mathrm{H}, \mathrm{H} 5-$ Ar2), 7.00 (d, $J=7.6 \mathrm{~Hz}, 1 \mathrm{H}, \mathrm{H} 4-\mathrm{Ar} 2), 2.33$ (s, 3H, $\left.\mathrm{CH}_{3}\right) ;{ }^{13} \mathrm{C} \mathrm{NMR}$ (125.8 MHz, DMSO- $\left.d_{6}\right) \delta 168.83\left(\mathrm{CO}_{2} \mathrm{H}\right), 158.32(2 \mathrm{C}=\mathrm{O}), 157.95$ $(1 \mathrm{C}=\mathrm{O}), 139.16$ (C2-Ar1), 137.92 (C3-Ar2), 137.31 (C1-Ar2), 134.24 (C4-Ar1), 131.44 (C6-Ar1), 128.50 (C5-Ar2), 125.44 (C4-Ar2), 123.94 (C5-Ar1), 121.06 (C2-Ar2), 119.53 (C3-Ar1), 117.82 (C6-Ar2), 117.31 (C1-Ar1), 21.12 (Me); ${ }^{15} \mathrm{~N}$ NMR (50.7 MHz, DMSO- $\left.d_{6}\right) \delta 126.9$ (N2), 121.8 (N1); IR $\nu$ (nujol): $\nu$ 3317, 3187, 1678, 1587, $1528 \mathrm{~cm}^{-1}$; HRMS (MALDI) calcd for $\mathrm{C}_{16} \mathrm{H}_{14} \mathrm{~N}_{2} \mathrm{O}_{4}[\mathrm{M}+\mathrm{Cs}]^{+} 431.0003$, found 431.0014; anal. calcd for $\mathrm{C}_{16} \mathrm{H}_{14} \mathrm{~N}_{2} \mathrm{O}_{4}$ : C, 64.42; H, 4.73; N, 9.39. Found: C, 64.31; H, 4.68; N, 9.42.

$N^{1}$-(2-Carboxyphenyl)- $N^{2}$-(4-methoxyphenyl)oxalamide (4d).

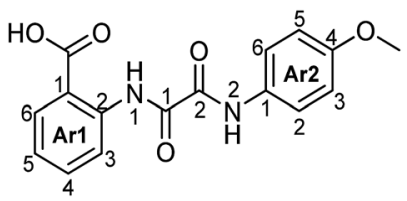

Grey powder $(0.50 \mathrm{~g}, 1.59 \mathrm{mmol}, 95 \%$ yield $): \operatorname{mp} 272-273{ }^{\circ} \mathrm{C}$; ${ }^{1} \mathrm{H}$ NMR (600 MHz, DMSO- $\left.d_{6}\right) \delta 12.70$ (s, $\left.1 \mathrm{H}, \mathrm{N} 1 \mathrm{H}\right), 10.78(\mathrm{~s}, 1 \mathrm{H}$, $\mathrm{N} 2 \mathrm{H}$ ), 8.69 (ddd, $J=8.4,7.7,1.0 \mathrm{~Hz}, 1 \mathrm{H}, \mathrm{H} 3-\mathrm{Ar} 1), 8.07$ (dd, $J=$ 8.2, 1.6 Hz, 1H, H6-Ar1), 7.78 (d, $J=9.1 \mathrm{~Hz}, 2 \mathrm{H}, \mathrm{H} 2,6-\mathrm{Ar} 2), 7.71$ 
(ddd, $J=7.9,7.8,1.6 \mathrm{~Hz}, 1 \mathrm{H}, \mathrm{H} 4-\mathrm{Ar} 1), 7.28$ (ddd, $J=7.8,7.4,1.1$ $\mathrm{Hz}, 1 \mathrm{H}, \mathrm{H} 5-\mathrm{Ar} 1$ ), 6.95 (d, $J=9.1 \mathrm{~Hz}, 2 \mathrm{H}, \mathrm{H} 3,5-\mathrm{Ar} 2), 3.76$ (s, 3H, $\left.\mathrm{OCH}_{3}\right) ;{ }^{13} \mathrm{C}$ NMR $\left(125.8 \mathrm{MHz}\right.$, DMSO- $\left.d_{6}\right) \delta 168.91\left(\mathrm{CO}_{2} \mathrm{H}\right), 158.56$ $(2 \mathrm{C}=\mathrm{O}), 157.66(1 \mathrm{C}=\mathrm{O}), 156.34$ (C4-Ar2), 139.24 (C2-Ar1), 134.31 (C4-Ar1), 131.51 (C6-Ar1), 130.54 (C1-Ar2), 124.01 (C5Ar1), 122.15 (C2-Ar2), 119.63 (C3-Ar1), 117.37 (C1-Ar1), 113.91 (C3-Ar2), 55.27 (OMe); ${ }^{15} \mathrm{~N}$ NMR (50.7 MHz, DMSO- $\left.d_{6}\right) \delta 125.6$ (N2), 121.8 (N1); IR (nujol): $\nu$ 3336, 3218, 1683, 1585, $1525 \mathrm{~cm}^{-1}$; HRMS (MALDI) calcd for $\mathrm{C}_{16} \mathrm{H}_{14} \mathrm{~N}_{2} \mathrm{O}_{5}[\mathrm{M}+\mathrm{Cs}]^{+} 446.9952$, found 446.9953; anal. calcd for $\mathrm{C}_{16} \mathrm{H}_{14} \mathrm{~N}_{2} \mathrm{O}_{5}$ : C, 61.14; $\mathrm{H}, 4.50 ; \mathrm{N}, 8.91$. Found: C, 60.99; H, 4.58; N, 8.97.

$N^{1}$-(2-Carboxyphenyl)- $N^{2}$-(3-nitrophenyl)oxalamide (4e).

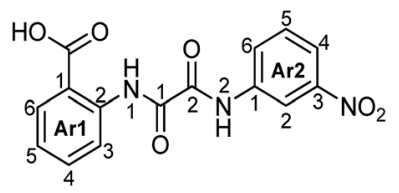

Brown powder $\left(0.53,1.61 \mathrm{mmol}, 96 \%\right.$ yield): $\mathrm{mp} 254-256{ }^{\circ} \mathrm{C}$; ${ }^{1} \mathrm{H}$ NMR (600 MHz, DMSO- $\left.d_{6}\right) \delta 12.90(\mathrm{~s}, 1 \mathrm{H}, \mathrm{N} 1 \mathrm{H}), 11.40(\mathrm{~s}, 1 \mathrm{H}$, $\mathrm{N} 2 \mathrm{H}$ ), 8.90 (dd, $J=2.0,2.0 \mathrm{~Hz}, 1 \mathrm{H}, \mathrm{H} 2-\mathrm{Ar} 2), 8.69$ (d, $J=8.2 \mathrm{~Hz}$, 1H, H3-Ar1), 8.27 (dd, $J=7.9,1.2 \mathrm{~Hz}, 1 \mathrm{H}, \mathrm{H} 6-\mathrm{Ar} 2$ ), 8.08 (dd, $J=$ 7.9, $1.2 \mathrm{~Hz}, 1 \mathrm{H}, \mathrm{H6}-\mathrm{Ar} 1$ ), 8.02 (dd, $J=7.9,1.8 \mathrm{~Hz}, 1 \mathrm{H}, \mathrm{H} 4-\mathrm{Ar} 2$ ), 7.66-7.72 (m, 2H, H5-Ar2; H4-Ar1), 7.28 (dd, $J=7.8,7.2 \mathrm{~Hz}, 1 \mathrm{H}$, H5-Ar1); ${ }^{13} \mathrm{C}$ NMR (150.9 MHz, DMSO- $\left.d_{6}\right) \delta 168.93\left(\mathrm{CO}_{2} \mathrm{H}\right), 158.76$ $(2 \mathrm{C}=\mathrm{O}), 157.68(1 \mathrm{C}=\mathrm{O}), 147.87$ (C3-Ar2), 139.05 (C2-Ar1), 138.71 (C1-Ar2), 134.02 (C4-Ar1), 131.43 (C6-Ar1), 130.09 (C5-Ar2), 126.68 (C6-Ar2), 123.97 (C5-Ar1), 119.51 (C3-Ar2), 119.17 (C4-Ar2), 117.80 (C1-Ar1), 114.81 (C2-Ar2); ${ }^{15} \mathrm{~N}$ NMR (60.8 MHz, DMSO- $\left.d_{6}\right) \delta 370.1$ $\left(\mathrm{NO}_{2}\right), 126.6$ (N2), 122.2 (N1); IR (nujol): $\nu$ 3326, 3188, 1690, 1589, $1532 \mathrm{~cm}^{-1}$; HRMS (MALDI) calcd for $\mathrm{C}_{15} \mathrm{H}_{11} \mathrm{~N}_{3} \mathrm{O}_{6}[\mathrm{M}+\mathrm{Na}]^{+}$ 352.0540, found 352.0563; anal. calcd for $\mathrm{C}_{15} \mathrm{H}_{11} \mathrm{~N}_{3} \mathrm{O}_{6}$ : C, 54.72; H, 3.37; N, 12.76. Found: C, 54.82; H, 3.43; N, 12.68 .

$N^{1}$-(2-Carboxyphenyl)- $N^{2}$-(4-ethylcarboxypheny)oxalamide (4f).

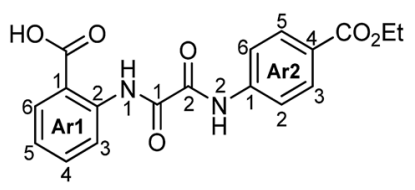

Light green powder (0.58 g, $1.63 \mathrm{mmol}, 97 \%$ yield): $\mathrm{mp} 268-$ $269{ }^{\circ} \mathrm{C} ;{ }^{1} \mathrm{H}$ NMR (600 MHz, DMSO- $d_{6}$ ) $\delta 12.78$ (s, $\left.1 \mathrm{H}, \mathrm{N} 1 \mathrm{H}\right), 11.18$ $(\mathrm{s}, 1 \mathrm{H}, \mathrm{N} 2 \mathrm{H}), 8.68$ (d, $J=8.8 \mathrm{~Hz}, 1 \mathrm{H}, \mathrm{H} 3-\mathrm{Ar} 1), 8.08$ (d, $J=7.7 \mathrm{~Hz}$, 1H, H6-Ar1), 8.04 (d, $J=8.8 \mathrm{~Hz}, 2 \mathrm{H}, \mathrm{H} 2,6-\mathrm{Ar} 2), 7.96$ (d, $J=8.8$ $\mathrm{Hz}, 2 \mathrm{H}, \mathrm{H} 3,5-\mathrm{Ar} 2$ ), 7.70 (dd, $J=7.7,7.7 \mathrm{~Hz}, 1 \mathrm{H}, \mathrm{H} 4-\mathrm{Ar} 1$ ), 7.28 (dd, $J=7.7,7.7 \mathrm{~Hz}, 1 \mathrm{H}, \mathrm{H} 5-A r 1), 4.30$ (q, $J=7.1 \mathrm{~Hz}, 2 \mathrm{H}$, $\mathrm{OCH}_{2} \mathrm{CH}_{3}$ ), $1.32\left(\mathrm{t}, J=7.1 \mathrm{~Hz}, 3 \mathrm{H}, \mathrm{OCH}_{2} \mathrm{CH}_{3}\right) ;{ }^{13} \mathrm{C}$ NMR (150.9 $\left.\mathrm{MHz}, \mathrm{DMSO}-d_{6}\right) \delta 168.90\left(\mathrm{CO}_{2} \mathrm{H}\right), 165.18\left(\mathrm{CO}_{2} \mathrm{Et}\right), 158.52(2 \mathrm{C}=$ $\mathrm{O}), 157.88(1 \mathrm{C}=\mathrm{O}), 141.74(\mathrm{C} 1-\mathrm{Ar} 2), 139.12$ (C2-Ar1), 134.17 (C4Ar1), 131.45 (C6-Ar1), 129.94 (C3-Ar2), 125.70 (C4-Ar2), 123.97 (C5-Ar1), 120.12 (C2-Ar2), 119.53 (C3-Ar1), 117.47 (C1-Ar1), 60.52 $\left(\mathrm{OCH}_{2} \mathrm{CH}_{3}\right), 14.13\left(\mathrm{OCH}_{2} \mathrm{CH}_{3}\right) ;{ }^{15} \mathrm{~N}$ NMR $\left(60.8 \mathrm{MHz}, \mathrm{DMSO}-d_{6}\right)$ $\delta 127.6$ (N2), 122.0 (N1); IR (nujol): $\nu$ 3355, 3260, 1708, 1688, 1602, 1586, 1524, 1271, $761 \mathrm{~cm}^{-1}$; HRMS (MALDI) calcd for $\mathrm{C}_{18} \mathrm{H}_{16} \mathrm{~N}_{2} \mathrm{O}_{6}[\mathrm{M}+2 \mathrm{Cs}-\mathrm{H}]^{+}$620.9034, found 620.9042; anal. calcd for $\mathrm{C}_{18} \mathrm{H}_{16} \mathrm{~N}_{2} \mathrm{O}_{6}$ : C, 60.67; H, 4.53; N, 7.86. Found: C, 60.59; $\mathrm{H}, 4.48$; N, 7.97 .
$N^{1}$-(4-Chloro-2-carboxyphenyl)- $N^{2}$-phenyloxalamide (4g).

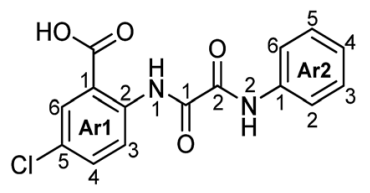

Brown powder (0.52 g, $1.63 \mathrm{mmol}, 98 \%$ yield): $\mathrm{mp} 242-243{ }^{\circ} \mathrm{C}$; ${ }^{1} \mathrm{H}$ NMR (600 MHz, DMSO- $\left.d_{6}\right) \delta 12.84(\mathrm{~s}, 1 \mathrm{H}, \mathrm{N} 1 \mathrm{H}), 10.89(\mathrm{~s}, 1 \mathrm{H}$, $\mathrm{N} 2 \mathrm{H}), 8.70$ (d, $J=8.9 \mathrm{~Hz}, 1 \mathrm{H}, \mathrm{H} 3-\mathrm{Ar} 1), 8.02$ (d, $J=2.6 \mathrm{~Hz}, 1 \mathrm{H}, \mathrm{H} 6-$ Ar1), 7.86 (d, $J=7.8 \mathrm{~Hz}, 2 \mathrm{H}, \mathrm{H} 2,6-\mathrm{Ar} 2), 7.76$ (dd, $J=8.9,2.6 \mathrm{~Hz}$, 1H, H4-Ar1), 7.39 (dd, $J=8.1,7.5 \mathrm{~Hz}, 2 \mathrm{H}, \mathrm{H} 3,5-\mathrm{Ar} 2), 7.18$ (dd, $J=$ 7.5, 7.4 Hz, $1 \mathrm{H}, \mathrm{H} 4-\mathrm{Ar} 2) ;{ }^{13} \mathrm{C} \mathrm{NMR}\left(100.9 \mathrm{MHz}\right.$, DMSO- $\left.d_{6}\right) \delta 167.59$ $\left(\mathrm{CO}_{2} \mathrm{H}\right), 158.34(2 \mathrm{C}=\mathrm{O}), 157.86(1 \mathrm{C}=\mathrm{O}), 137.98(\mathrm{C} 2-\mathrm{Ar} 1), 137.37$ (C1-Ar2), 133.58 (C4-Ar1), 130.61 (C6-Ar1), 128.64 (C3-Ar2), 127.51 (C5-Ar1), 124.74 (C4-Ar2), 121.29 (C3-Ar1), 120.61 (C2-Ar2), 119.71 (C1-Ar1); ${ }^{15} \mathrm{~N}$ NMR (50.7 MHz, DMSO- $\left.d_{6}\right) \delta 127.0$ (N2), 121.4 (N1); IR (nujol): $\nu$ 3331, 3166, 1679, 1579, $1505 \mathrm{~cm}^{-1}$; HRMS (MALDI) calcd for $\mathrm{C}_{15} \mathrm{H}_{11} \mathrm{ClN}_{2} \mathrm{O}_{4}[\mathrm{M}+\mathrm{Na}]^{+}$341.0300, found 341.0328; anal. calcd for $\mathrm{C}_{15} \mathrm{H}_{11} \mathrm{ClN}_{2} \mathrm{O}_{4}$ : C, 56.53; H, 3.48; Cl, 11.12; N, 8.79. Found: C, 56.59; H, 3.52; Cl, 11.07; N, 8.99.

$\mathrm{N}$-(2-Carboxyphenyl)oxalamide (4h).

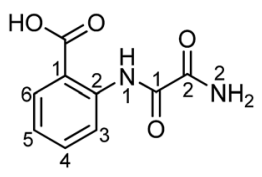

Violet powder (0.34 g, $1.62 \mathrm{mmol}, 97 \%$ yield): $\mathrm{mp} 267-268{ }^{\circ} \mathrm{C}$; ${ }^{1} \mathrm{H}$ NMR (600 MHz, DMSO- $d_{6}$ ) $\delta 12.51(\mathrm{~s}, 1 \mathrm{H}, \mathrm{N} 1 \mathrm{H}), 8.65$ (d, $J=8.2$ Hz, 1H, H3-Ar), 8.36 (br.s, $1 \mathrm{H}, \mathrm{NH}_{2}$ ), 8.05 (br.s, $1 \mathrm{H}, \mathrm{NH}_{2}$ ), 8.04 (dd, $J$ $=8.0,1.6 \mathrm{~Hz}, 1 \mathrm{H}, \mathrm{H6}-\mathrm{Ar}$ ), 7.68 (ddd, $J=7.9,7.9,1.5 \mathrm{~Hz}, 1 \mathrm{H}, \mathrm{H} 4-\mathrm{Ar}$ ), 7.25 (ddd, $J=7.6,7.6,1.0 \mathrm{~Hz}, 1 \mathrm{H}, \mathrm{H} 5-\mathrm{Ar}) ;{ }^{13} \mathrm{C} \mathrm{NMR}(125.8 \mathrm{MHz}$, DMSO- $\left.d_{6}\right) \delta 168.66\left(\mathrm{CO}_{2} \mathrm{H}\right), 161.57(1 \mathrm{C}=\mathrm{O}), 158.73(2 \mathrm{C}=\mathrm{O}), 139.19$ (C2-Ar), 134.13 (C4-Ar), 131.38 (C6-Ar), 123.70 (C5-Ar), 119.47 (C3Ar), 117.22 (C1-Ar); ${ }^{15} \mathrm{~N}$ NMR (50.7 MHz, DMSO- $\left.d_{6}\right) \delta 103.5$ (N2), 121.3 (N1); IR (nujol): $\nu$ 3538, 3465, 3321, 3168, 1729, 1683, 1592, 1537, 1272, $752 \mathrm{~cm}^{-1}$; HRMS (MALDI) calcd for $\mathrm{C}_{9} \mathrm{H}_{8} \mathrm{~N}_{2} \mathrm{O}_{4}[\mathrm{M}+$ $2 \mathrm{Cs}-\mathrm{H}]^{+}$472.8509, found 472.8491; anal. calcd for $\mathrm{C}_{9} \mathrm{H}_{8} \mathrm{~N}_{2} \mathrm{O}_{4}: \mathrm{C}$, 51.93; H, 3.87; N, 13.46. Found: C, 51.90; H, 3.89; N, 13.42.

Synthesis of (3-(2-nitroaryl)oxiran-2-yl)(aryl)methanones (6a-d). Synthesis of (3-(2-nitroaryl)oxiran-2-yl)(aryl)methanones (6a-d) was performed according to the general procedure for the Darzens condensation with the use of corresponding (halomethyl)arylketones (5a-c) instead of chloroacetamides $(\mathbf{2 a}-\mathbf{g})$. In these cases as distinct from the synthesis of 3-(2-nitroaryl) oxiran-2-carboxamides $(\mathbf{3 a}-\mathbf{h})$ the reactions are completed for $0.5 \mathrm{~h}$. The products precipitated during the reaction and did not require any purification except washing with water $(3 \times 25 \mathrm{~mL})$. trans-(3-(2-Nitrophenyl)oxiran-2-yl)(4-tolyl)methanone (6a).

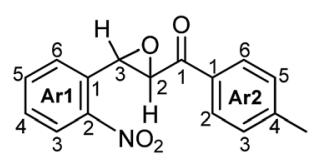

White powder (7.65 g, $0.027 \mathrm{~mol}, 100 \%$ yield): $\mathrm{mp} 154{ }^{\circ} \mathrm{C} ;{ }^{1} \mathrm{H}$ NMR (400 MHz, DMSO- $d_{6}$ ) $\delta 8.21$ (dd, $J=8.1,1.0 \mathrm{~Hz}, 1 \mathrm{H}, \mathrm{H} 3-$ 
Ar1), 8.0 (d, $J=8.2 \mathrm{~Hz}, 2 \mathrm{H}, \mathrm{H} 3,5-\mathrm{Ar} 2), 7.88$ (ddd, $J=7.6,7.5,0.8$ $\mathrm{Hz}, 1 \mathrm{H}, \mathrm{H} 5-\mathrm{Ar} 1), 7.65-7.71$ (m, 2H, H4,6-Ar1), 7.37 (d, $J=8.2 \mathrm{~Hz}$, 2H, H2,6-Ar1), 4.74 (d, $J=2.1 \mathrm{~Hz}, 1 \mathrm{H}, \mathrm{H} 2$ ), 4.56 (d, $J=2.1 \mathrm{~Hz}$, 1H, H3), 2.41 (s, 3H, Me); ${ }^{13} \mathrm{C}$ NMR (100.6 MHz, DMSO- $\left.d_{6}\right)$ $\delta 192.14(1 \mathrm{C}=\mathrm{O}), 147.47$ (C2-Ar1), 144.72 (C4-Ar2), 134.60 (C5Ar1), 132.70 (C1-Ar2), 132.42 (C1-Ar1), 129.47 (C4-Ar1), 129.38 (C2-Ar2), 128.50 (C3-Ar2), 127.02 (C6-Ar1), 124.62 (C3-Ar1), 58.34 (C2), 57.15 (C3), 21.19 (Me). IR (nujol): $\nu$ 1682, $1522 \mathrm{~cm}^{-1}$; HRMS (MALDI) calcd for $\mathrm{C}_{16} \mathrm{H}_{13} \mathrm{NO}_{4}[\mathrm{M}+\mathrm{Cs}]^{+} 415.9894$, found 415.9895; anal. calcd for $\mathrm{C}_{16} \mathrm{H}_{13} \mathrm{NO}_{4}$ : C, 67.84; $\mathrm{H}, 4.63 ; \mathrm{N}, 4.94$; found: C, 67.89; H, 4.62; N, 4.98.

trans-(4-Methoxyphenyl)(3-(2-nitrophenyl)oxiran-2-yl)methanone $(6 b)$.

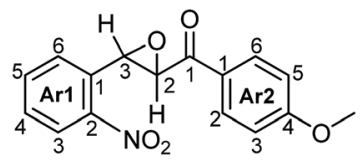

White powder (8.08 g, $0.027 \mathrm{~mol}, 100 \%$ yield): $\mathrm{mp} 141{ }^{\circ} \mathrm{C} ;{ }^{1} \mathrm{H}$ NMR $\left(400 \mathrm{MHz}, \mathrm{DMSO}-d_{6}\right) \delta 8.21$ (d, $\left.J=8.2 \mathrm{~Hz}, 1 \mathrm{H}, \mathrm{H} 3-\mathrm{Ar} 1\right), 8.09$ (d, $J=$ $8.1 \mathrm{~Hz}, 2 \mathrm{H}, \mathrm{H} 2,6-\mathrm{Ar} 2), 7.87$ (dd, $J=7.6,7.4 \mathrm{~Hz}, 1 \mathrm{H}, \mathrm{H} 5-\mathrm{Ar} 1), 7.65-$ 7.71 (m, 2H, H6,4-Ar1), 7.08 (d, $J=8.1 \mathrm{~Hz}, 2 \mathrm{H}, \mathrm{H} 3,5-\mathrm{Ar} 2), 4.71$ (d, $J$ $=1.7 \mathrm{~Hz}, 1 \mathrm{H}, \mathrm{H} 2), 4.56(\mathrm{~d}, J=1.7 \mathrm{~Hz}, 1 \mathrm{H}, \mathrm{H} 3), 3.87$ (s, 3H, OMe); ${ }^{13} \mathrm{C}$ NMR (125.8 MHz, DMSO- $\left.d_{6}\right) \delta 190.82(1 \mathrm{C}=\mathrm{O}), 163.87$ (C4-Ar2), 147.45 (C2-Ar1), 134.60 (C5-Ar1), 132.53 (C1-Ar1), 130.87 (C2-Ar2), 129.43 (C4-Ar1), 128.20 (C1-Ar2), 127.05 (C6-Ar1), 124.61 (C3-Ar1), 114.11 (C3-Ar2), 58.17 (C2), 57.03 (C3), 55.58 (OMe). IR (nujol): $\nu$ 1678, 1597, 1513; HRMS (MALDI) calcd for $\mathrm{C}_{16} \mathrm{H}_{13} \mathrm{NO}_{5}[\mathrm{M}+\mathrm{Cs}]^{+}$ 431.9843, found 431.9813; anal. calcd for $\mathrm{C}_{16} \mathrm{H}_{13} \mathrm{NO}_{5}$ : C, 64.21; $\mathrm{H}$, 4.38; N, 4.68; found: C, 64.39; H, 4.51; N, 4.77.

trans-(3-Nitrophenyl)(3-(2-nitrophenyl)oxiran-2-yl)methanone (6c).

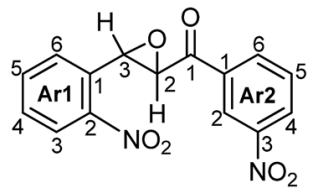

Grey powder (8.49 g, $0.027 \mathrm{~mol}, 100 \%$ yield): $\mathrm{mp} 156-157{ }^{\circ} \mathrm{C} ;{ }^{1} \mathrm{H}$ NMR (400 MHz, DMSO- $d_{6}$ ) $\delta 8.79$ (s, 1H, H2-Ar2), 8.53 (d, $J=8.2$ $\mathrm{Hz}, 2 \mathrm{H}, \mathrm{H} 4,6-\mathrm{Ar} 2$ ), 8.23 (d, $J=8.0 \mathrm{~Hz}, 1 \mathrm{H}, \mathrm{H} 3-\mathrm{Ar} 1$ ), 7.86-7.11 (m, 2H, H5-Ar1, H5-Ar2), 7.67-7.72 (m, 2H, H4,6-Ar1), 4.90 (d, J = 1.9 $\mathrm{Hz}, 1 \mathrm{H}, \mathrm{H} 2), 4.65$ (d, $J=1.9 \mathrm{~Hz}, 1 \mathrm{H}, \mathrm{H} 3) ;{ }^{13} \mathrm{C} \mathrm{NMR}(125.8 \mathrm{MHz}$, DMSO-d $\left.{ }_{6}\right) \delta 191.77(1 \mathrm{C}=\mathrm{O}), 148.05$ (C3-Ar2), 147.45 (C2-Ar1), 136.05 (C1-Ar2), 134.70 (C5-Ar1), 134.63 (C6-Ar2), 132.15 (C1Ar1), 130.72 (C5-Ar2), 129.64 (C4-Ar1), 128.17 (C4-Ar2), 127.07 (C6-Ar1), 124.68 (C3-Ar1), 122.71 (C2-Ar2), 58.71 (C2), 57.73 (C3). IR (nujol): $\nu 1697,1522 \mathrm{~cm}^{-1}$; HRMS (MALDI) calcd for $\mathrm{C}_{15} \mathrm{H}_{10} \mathrm{~N}_{2} \mathrm{O}_{6}$ $[\mathrm{M}+\mathrm{Cs}]^{+} 446.9588$, found 446.9581; anal. calcd for $\mathrm{C}_{15} \mathrm{H}_{10} \mathrm{~N}_{2} \mathrm{O}_{6}: \mathrm{C}$, 57.28; H, 3.32; N, 8.78; found: C, 57.33; H, 3.21; N, 8.91.

trans-(3-(5-Chloro-2-nitrophenyl)oxiran-2-yl)(4-tolyl)methanone (6d).

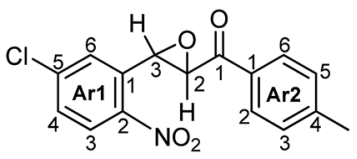

Tan powder (8.58 g, $0.027 \mathrm{~mol}, 100 \%$ yield): $\operatorname{mp~} 139{ }^{\circ} \mathrm{C} ;{ }^{1} \mathrm{H}$ NMR (400 MHz, DMSO- $d_{6}$ ) $\delta 8.24$ (d, $J=8.8 \mathrm{~Hz}, 1 \mathrm{H}, \mathrm{H} 3-\mathrm{Ar} 1$ ), 8.01 (d, $J=8.1 \mathrm{~Hz}, 2 \mathrm{H}, \mathrm{H} 2,6-\mathrm{Ar} 2), 7.75$ (dd, $J=8.8,2.4 \mathrm{~Hz}$, 1H, H4-Ar1), 7.59 (d, $J=2.4 \mathrm{~Hz}, 1 \mathrm{H}, \mathrm{H} 6-\mathrm{Ar} 1), 7.37$ (d, $J=8.1 \mathrm{~Hz}$, $2 \mathrm{H}, \mathrm{H} 3,5-\mathrm{Ar} 2), 4.81$ (d, $J=2.1 \mathrm{~Hz}, 1 \mathrm{H}, \mathrm{H} 2), 4.60$ (d, $J=2.1 \mathrm{~Hz}$, $1 \mathrm{H}, \mathrm{H} 3), 2.41$ (s, 3H, Me); ${ }^{13} \mathrm{C}$ NMR (125.8 MHz, DMSO- $d_{6}$ ) $\delta 191.86(1 \mathrm{C}=\mathrm{O}), 146.17$ (C2-Ar1), 144.85 (C4-Ar2), 139.44 (C5Ar1), 134.86 (C1-Ar1), 132.64 (C1-Ar2), 129.41 (C3-Ar2), 129.35 (C4-Ar1), 128.56 (C2-Ar2), 126.82 (C3-Ar1), 126.71 (C6-Ar1), 58.24 (C2), 56.65 (C3), 21.20 (Me). IR (nujol): $\nu$ 1681, 1607, 1516; HRMS (MALDI) calcd for $\mathrm{C}_{16} \mathrm{H}_{12} \mathrm{ClNO}_{4}[\mathrm{M}+\mathrm{Cs}]^{+}$449.9504, found 449.9522; anal. calcd for $\mathrm{C}_{16} \mathrm{H}_{12} \mathrm{ClNO}_{4}$ : C, 60.48; H, 3.81; Cl, 11.16; N, 4.41; found: C, 60.31; H, 3.71; N, 4.61.

Synthesis of 2-(2-oxo-2-arylacetamido)benzoic acids (7a-d). Synthesis of 2-(2-oxo-2-arylacetamido)benzoic acids (7a-d) was performed according to the general procedure for the rearrangement with the use of corresponding (3-(2-nitroaryl)oxiran2-yl)(aryl)methanones (6a-d) instead of 3-(2-nitroaryl)oxiran-2carboxamides $(\mathbf{2 a - h})$. Products $\mathbf{7 a}, \mathbf{b}$ were purified by washing with acetone $(3 \times 1 \mathrm{~mL})$, products $\mathbf{7 c , d}-$ by recrystallization from $\mathrm{AcOH}$.

2-[2-Oxo-2-(4-tolyl)acetamido]benzoic acid (7a).

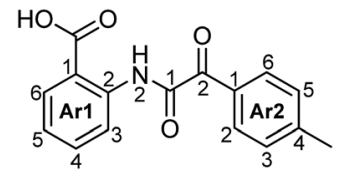

Brown powder (0.35 g, $1.25 \mathrm{mmol}$, 75\% yield): mp 199-201 ${ }^{\circ} \mathrm{C} ;{ }^{1} \mathrm{H}$ NMR $\left(400 \mathrm{MHz}, \mathrm{DMSO}-d_{6}\right) \delta 12.72(\mathrm{~s}, 1 \mathrm{H}, \mathrm{NH}), 8.64(\mathrm{~d}, J=$ $7.5 \mathrm{~Hz}, 1 \mathrm{H}, \mathrm{H} 3-\mathrm{Ar} 1$ ), 8.14 (d, $J=7.8 \mathrm{~Hz}, 2 \mathrm{H}, \mathrm{H} 2,6-\mathrm{Ar} 2), 8.04$ (dd, $J$ $=8.1,1.0 \mathrm{~Hz}, 1 \mathrm{H}, \mathrm{H} 6-\mathrm{Ar} 1), 7.71$ (dd, $J=7.6,7.5 \mathrm{~Hz}, 1 \mathrm{H}, \mathrm{H} 4-\mathrm{Ar} 1)$, 7.37 (d, $J=7.8 \mathrm{~Hz}, 2 \mathrm{H}, \mathrm{H} 3,5-\mathrm{Ar} 2), 7.28(\mathrm{dd}, J=7.5,7.9 \mathrm{~Hz}, 1 \mathrm{H}$, H5-Ar1), 2.42 (s, 3H, Me); ${ }^{13} \mathrm{C}$ NMR (125.8 MHz, DMSO- $d_{6}$ ) $\delta 187.00(2 \mathrm{C}=\mathrm{O}), 169.15\left(\mathrm{CO}_{2} \mathrm{H}\right), 160.57(1 \mathrm{C}=\mathrm{O}), 145.42(\mathrm{C} 4-$ Ar2), 139.28 (C2-Ar1), 134.19 (C4-Ar1), 131.41 (C6-Ar1), 130.94 (C2-Ar2), 130.38 (C1-Ar2), 129.27 (C3-Ar2), 124.00 (C5-Ar1), 120.13 (C3-Ar1), 117.75 (C1-Ar1), 21.39 (Me). IR (nujol): $\nu$ 3363, 1678, 1604, 1586, 1521, $1262 \mathrm{~cm}^{-1}$; HRMS (MALDI) calcd for $\mathrm{C}_{16} \mathrm{H}_{13} \mathrm{NO}_{4}[\mathrm{M}+\mathrm{Cs}]^{+} 415.9894$, found 415.9887; anal. calcd for $\mathrm{C}_{16} \mathrm{H}_{13} \mathrm{NO}_{4}$ : C, 67.84; H, 4.63; N, 4.94. Found: C, 67.92; H, 4.68; $\mathrm{N}, 4.71$.

2-[2-(4-Methoxyphenyl)-2-oxoacetamido]benzoic acid (7b).

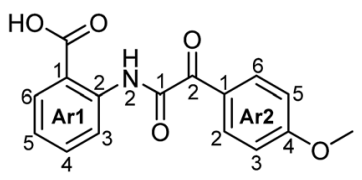

Brown powder (0.40 g, $1.35 \mathrm{mmol}$, 81\% yield): mp 178-181 ${ }^{\circ} \mathrm{C} ;{ }^{1} \mathrm{H}$ NMR $\left(400 \mathrm{MHz}, \mathrm{DMSO}-d_{6}\right) \delta 12.37(\mathrm{~s}, 1 \mathrm{H}, \mathrm{NH}), 8.65(\mathrm{~d}, J=$ $8.3 \mathrm{~Hz}, 1 \mathrm{H}, \mathrm{H} 3-\mathrm{Ar} 1$ ), 8.27 (d, $J=8.9 \mathrm{~Hz}, 2 \mathrm{H}, \mathrm{H} 2,6-\mathrm{Ar} 2), 8.05$ (d, $J$ $=8.0 \mathrm{~Hz}, 1 \mathrm{H}, \mathrm{H} 6-\mathrm{Ar} 1$ ), 7.67 (dd, $J=7.2,8.3 \mathrm{~Hz}, 1 \mathrm{H}, \mathrm{H} 4-\mathrm{Ar} 1), 7.26$ (dd, $J=7.2,7.8 \mathrm{~Hz}, 1 \mathrm{H}, \mathrm{H} 5-\mathrm{Ar} 1$ ), 8.10 (d, $J=8.9 \mathrm{~Hz}, 2 \mathrm{H}, \mathrm{H} 3,5-$ Ar2), 3.88 (s, 3H, OMe); ${ }^{13} \mathrm{C}$ NMR (125.8 MHz, DMSO- $\left.d_{6}\right)$ $\delta 185.42(1 \mathrm{C}=\mathrm{O}), 169.12\left(\mathrm{CO}_{2} \mathrm{H}\right), 164.39(\mathrm{C} 4-\mathrm{Ar} 2), 160.81(2 \mathrm{C}=$ O), 139.35 (C2-Ar1), 134.14 (C4-Ar1), 133.47 (C2-Ar2), 131.39 (C6- 
Ar1), 125.61 (C4-Ar2), 123.88 (C5-Ar1), 120.04 (C3-Ar1), 117.60 (C1-Ar1), 114.10 (C3-Ar2), 55.70 (OMe). IR (nujol): $\nu$ 3472, 3258, 1702, 1674, 1601, 1584, 1528, $1260 \mathrm{~cm}^{-1}$; HRMS (MALDI) calcd for $\mathrm{C}_{16} \mathrm{H}_{13} \mathrm{NO}_{5}[\mathrm{M}+\mathrm{Cs}]^{+} 431.9843$, found 431.9828; anal. calcd for $\mathrm{C}_{16} \mathrm{H}_{13} \mathrm{NO}_{5}$ : C, 64.21; $\mathrm{H}, 4.38 ; \mathrm{N}, 4.68$. Found: $\mathrm{C}, 64.32 ; \mathrm{H}$, $4.37 ; \mathrm{N}, 4.75$.

2-[2-(3-Nitrophenyl)-2-oxoacetamido]benzoic acid (7c).

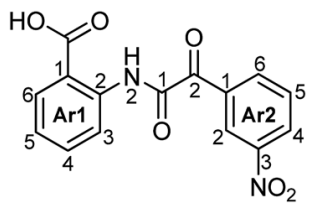

Brown powder (0.45 g, $1.44 \mathrm{mmol}, 86 \%$ yield): mp 251-252 ${ }^{\circ} \mathrm{C} ;{ }^{1} \mathrm{H}$ NMR (400 MHz, DMSO- $d_{6}$ ) $\delta 12.60$ (s, 1H, NH), 9.06 (s, $1 \mathrm{H}, \mathrm{H} 2-\mathrm{Ar} 2), 8.70$ (d, $J=8.1 \mathrm{~Hz}, 1 \mathrm{H}, \mathrm{H} 3-\mathrm{Ar} 1), 8.58$ (d, $J=7.7 \mathrm{~Hz}$, 1H, H6-Ar2), 8.54 (dd, $J=8.2,1.5 \mathrm{~Hz}, 1 \mathrm{H}, \mathrm{H} 4-\mathrm{Ar} 2$ ), 8.09 (dd, $J=$ 7.9, $1.5 \mathrm{~Hz}, 1 \mathrm{H}, \mathrm{H6}-\mathrm{Ar} 1$ ), 7.89 (dd, $J=7.9,8.0 \mathrm{~Hz}, 1 \mathrm{H}, \mathrm{H} 5-\mathrm{Ar} 2$ ), 7.72 (ddd, $J=7.9,7.9,1.5 \mathrm{~Hz}, 1 \mathrm{H}, \mathrm{H} 4-\mathrm{Ar} 1), 7.30$ (dd, $J=7.9,7.9$ $\mathrm{Hz}, 1 \mathrm{H}, \mathrm{H} 5-\mathrm{Ar} 1) ;{ }^{13} \mathrm{C} \mathrm{NMR}\left(125.8 \mathrm{MHz}, \mathrm{DMSO}-d_{6}\right) \delta 185.04(1 \mathrm{C}=$ $\mathrm{O}), 169.14\left(\mathrm{CO}_{2} \mathrm{H}\right), 159.14(2 \mathrm{C}=\mathrm{O}), 147.38(\mathrm{C} 3-\mathrm{Ar} 2), 139.26(\mathrm{C} 2-$ Ar1), 136.71 (C6-Ar2), 134.46 (C1-Ar2), 134.22 (C4-Ar1), 131.44 (C6-Ar1), 130.14 (C5-Ar2), 128.038 (C4-Ar2), 125.58 (C2-Ar2), 123.99 (C5-Ar1), 119.88 (C3-Ar1), 117.58 (C1-Ar1). IR (nujol): $\nu$ 3413, 3189, 1695, 1679, 1588, 1532, $1288 \mathrm{~cm}^{-1}$; HRMS (MALDI) calcd for $\mathrm{C}_{15} \mathrm{H}_{10} \mathrm{~N}_{2} \mathrm{O}_{6}[\mathrm{M}+2 \mathrm{Cs}-\mathrm{H}]^{+}$578.8564, found 578.8545; anal. calcd for $\mathrm{C}_{15} \mathrm{H}_{10} \mathrm{~N}_{2} \mathrm{O}_{6}: \mathrm{C}, 57.28 ; \mathrm{H}, 3.32 ; \mathrm{N}, 8.78$; found: $\mathrm{C}$, $57.34 ; \mathrm{H}, 3.37$; N, 8.89.

5-Chloro-2-[2-oxo-2-(4-tolyl)acetamido]benzoic acid (7d).

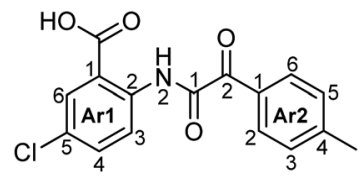

Light yellow powder (0.47 g, $1.49 \mathrm{mmol}, 89 \%$ yield): $\mathrm{mp} 257-$ $258{ }^{\circ} \mathrm{C} ;{ }^{1} \mathrm{H}$ NMR $\left(400 \mathrm{MHz}, \mathrm{DMSO}-d_{6}\right) \delta 12.30(\mathrm{~s}, 1 \mathrm{H}, \mathrm{NH}), 8.64$ (d, $J=8.9 \mathrm{~Hz}, 1 \mathrm{H}, \mathrm{H} 3-\mathrm{Ar} 1), 8.13$ (d, $J=7.9 \mathrm{~Hz}, 2 \mathrm{H}, \mathrm{H} 2,6-\mathrm{Ar} 2)$, 7.97 (br.s, 1H, H6-Ar1), 7.74 (dd, $J=8.8,1.8 \mathrm{~Hz}, 1 \mathrm{H}, \mathrm{H} 4-\mathrm{Ar} 1$ ), 7.38 (d, $J=7.9, \mathrm{~Hz}, 2 \mathrm{H}, \mathrm{H} 3,5-\mathrm{Ar} 2), 2.41$ (s, 3H, Me); ${ }^{13} \mathrm{C} \mathrm{NMR}$ $\left(125.8 \mathrm{MHz}, \mathrm{DMSO}-d_{6}\right) \delta 186.46(1 \mathrm{C}=\mathrm{O}), 167.80\left(\mathrm{CO}_{2} \mathrm{H}\right), 160.35$ $(2 \mathrm{C}=\mathrm{O}), 145.35$ (C4-Ar2), 138.03 (C2-Ar1), 133.70 (C4-Ar1), 130.90 (C2-Ar2), 130.51 (C6-Ar1), 130.25 (C1-Ar2), 129.16 (C3Ar2), 127.55 (C5-Ar1), 121.83 (C3-Ar1), 119.53 (C1-Ar1), 21.32 (Me). IR (nujol): $\nu$ 3219, 1703, 1681, 1598, 1577, 1521, $1248 \mathrm{~cm}^{-1}$; HRMS (MALDI) calcd for $\mathrm{C}_{16} \mathrm{H}_{12} \mathrm{ClNO}_{4}[\mathrm{M}+2 \mathrm{Cs}-\mathrm{H}]^{+} 581.8480$, found 581.8455; anal. calcd for $\mathrm{C}_{16} \mathrm{H}_{12} \mathrm{ClNO}_{4}$ : C, 60.48; H, 3.81; Cl, 11.16; N, 4.41; found: C, 60.52; H, 3.73; N, 4.58.

\section{Conflict of interest}

The authors declare no competing financial interest.

\section{Acknowledgements}

This work was partially supported by the Russian scientific foundation (grants No. 14-23-00073 and No. 14-50-00014).

\section{References}

1 (a) J. G. Smith, Synthesis, 1984, 629; (b) E. G. Lewars, in Comprehensive Heterocyclic Chemistry, ed. A. R. Katritzky and C. W. Rees, Pergamon, Oxford, 1984, vol. 7, p. 95; (c) M. Bartok and K. L. Lang, in The Chemistry of Heterocyclic Compounds: Small Ring Heterocycles, Part 3, ed. A. Hassner, Wiley Intersience, New York, 1983, vol. 42, p. 1.

2 A. S. Rao, S. K. Paknikar and J. G. Kirtane, Tetrahedron, 1983, 39, 2323.

3 (a) B. Rickborn, in Comprehensive Organic Synthesis, ed. B. M. Trost, Pergamon, Oxford, 1991, vol. 3, p. 733; (b) K. Maruoka, N. Murase, R. Bureau, T. Ooi and H. Yamamoto, Tetrahedron, 1994, 50, 3663; (c) H. O. House, J. Am. Chem. Soc., 1955, 77, 3070.

4 R. E. Parker and N. S. Isaacs, Chem. Rev., 1959, 59, 737.

5 B. E. Rossiter, in Asymmetric Synthesis, ed. J. D. Morrison, Academic Press, Orlando, FL, 1983, vol. 5, p. 194.

6 L. S. Hegedus, Transition Metals in the Synthesis of Complex Organic Molecules, University Science Books, Menlo Park, CA, 1994.

7 (a) G. Adames, C. Bibby and R. Grigg, J. Chem. Soc., Chem. Commun., 1972, 491; (b) D. Milstein, O. Buchman and J. Blum, J. Org. Chem., 1977, 42, 2299; (c) D. Milstein, J. Am. Chem. Soc., 1982, 104, 5227.

8 (a) M. Suzuki, Y. Oda and R. Noyori, J. Am. Chem. Soc., 1979, 101, 1623; (b) M. Suzuki, A. Watanabe and R. Noyori, J. Am. Chem. Soc., 1980, 102, 2095; (c) T. Hirao, N. Yamada, Y. Ohshiro and T. Agawa, Chem. Lett., 1982, 1997; (d) Y. D. Vankar, N. C. Chaudhuri and S. P. Singh, Synth. Commun., 1986, 16, 1621; (e) G. Visentin, O. Piccolo and G. Consiglio, J. Mol. Catal., 1990, 61, L1; (f) S. Kulasegaram and R. J. Kulawiec, J. Org. Chem., 1997, 62, 6547.

9 H. Alper, D. Roches, T. Durst and R. Legault, J. Org. Chem., 1976, 41, 3611.

10 J. Prandi, J. L. Namy, G. Menoret and H. B. Kagan, J. Organomet. Chem., 1985, 285, 449.

11 (a) R. Aumann, K. Frohlich and H. Ring, Angew. Chem., Int. Ed., 1974, 13, 275; (b) K. Hayakawa and H. Schmid, Helv. Chim. Acta, 1977, 60, 1942; (c) T. Kauffmann, C. Neiteler and G. Neiteler, Chem. Ber., 1994, 127, 659.

12 B. C. Ranu and U. Jana, J. Org. Chem., 1998, 63, 8212.

13 V. A. Mamedov, V. L. Mamedova, S. F. Kadyrova, G. Z. Khikmatova, A. T. Gubaidullin, I. K. Rizvanov and S. K. Latypov, Tetrahedron, 2015, 71, 2670.

14 W.-J. Liu, B.-D. Lv and L.-Z. Gong, Angew. Chem., Int. Ed., 2009, 48, 6503.

15 (a) A. E. Derome, Modern NMR Techniques for Chemistry Research, Pergamon, Cambridge, UK, 1988; (b) T. I. Atta-urRahman, One and Two Dimensional NMR Spectroscopy, Elsevier, Amsterdam, 1989, p. 578.

16 L. Shi, R. Hu, Y. Wei, Y. Liang, Z. Yang and S. Ke, Eur. J. Med. Chem., 2012, 54, 549.

17 D. Merk, M. Gabler, R. Carrasco Gomez, D. Flesch, T. Hanke, A. Kaiser, C. Lamers, O. Werz, G. Schneider and M. SchubertZsilavecz, Bioorg. Med. Chem., 2014, 22, 2447. 
18 (a) A. Z. Bialvaei and H. S. Kafil, Curr. Med. Res. Opin., 2015, 31, 707; (b) A. Kristiansen, M. Grgic, B. Altermark and I. Leiros, J. Antimicrob. Chemother., 2015, 70, 766; (c) F. J. Barreyro, S. Holod, P. V. Finocchietto, A. M. Camino, J. B. Aquino, A. Avagnina, M. C. Carreras, J. J. Poderoso and G. Gores, Liver Int., 2015, 35, 953; (d) P. K. Sahoo and P. Behera, Eur. J. Med. Chem., 2010, 45, 909; (e) F. D. Settimo, J. Med. Chem., 2001, 44, 4359; (f) A. Michel, P. Downey, J. M. Nicolas and D. Scheller, PLoS One, 2014, 9, e114086; $(g)$ G. Barta-Szalai, I. Borza and E. Bozo, Bioorg. Med. Chem. Lett., 2004, 14, 3953.

19 K. O. Yerdelen, Med. Chem. Res., 2015, 24, 588.

20 T. J. Lanza, P. L. Durette, T. Rollins, S. Siciliano, D. N. Cianciarulo, S. V. Kobayashi, C. G. Caldwell, M. S. Springer and W. K. Hagmann, J. Med. Chem., 1992, 35, 252.

21 H. J. Zhong, L. J. Liu and C. M. Chong, PLoS One, 2014, 9, e92905.

22 (a) H. Otsuki, T. Hishiki, T. Miura, C. Hashimoto, T. Narumi, H. Tamamura, K. Yoshimura, S. Matsushita and T. J. Igarashi, J. Gen. Virol., 2013, 94, 2710; (b) C. Hashimoto, T. Narumi and H. Otsuki, Bioorg. Med. Chem., 2013, 21, 7884; (c) T. Narumi, H. Arai, K. Yoshimura, S. Harada, W. Nomura, S. Matsushita and H. Tamamura, Bioorg. Med. Chem., 2011, 19, 6735.

23 A. P. Nikalje, M. Ghodke and A. Girbane, Arch. Pharmacol., 2012, 345, 57.

24 (a) S. B. Singh, F. Pelaez, D. J. Hazuda and R. B. Lingham, Drugs Future, 2005, 30, 277; (b) J. P. Guare, J. S. Wai, R. P. Gomez, N. J. Anthony, S. M. Jolly, A. R. Cortes, J. P. Vacca, P. J. Felock, K. A. Stillmock, W. A. Schleif, G. Moyer, L. J. Gabryelski, L. Jin, I. W. Chen, D. J. Hazuda and S. D. Young, Bioorg. Med. Chem. Lett., 2006, 16, 2900; (c) T. W. North, A. Villalobos and S. J. Hurwitz, Antimicrob. Agents Chemother., 2014, 58, 3927.

25 (a) P. K. Jadhav and H. W. Man, Tetrahedron Lett., 1996, 37, 1153-1156; (b) M. Medow, G. Priem, G. Quelever, M. Camplo and J. K. Kraus, Tetrahedron Lett., 1998, 39, 4021; (c) Q. Zhao, L. Ma, S. Jiang, H. Lu, S. Liu, Y. He, N. Strick, N. Neamati and A. K. Debnath, Virology, 2005, 339, 213; (d) F. Curreli, S. Choudhury, I. Pyatkin, V. P. Zagorodnikov, A. K. Bulay, A. Altieri, Y. D. Kwon, P. D. Kwong and A. K. Depnath, J. Med. Chem., 2012, 55, 4764-4775.

26 U. D. Treuner and H. Breuer, US Pat., 4, 1978, 113, 943, 1978transChem. Abstr., 1979, 90, 72212.

27 J. J. Hale, S. G. Mills, M. MacCoss, P. E. Finke, M. A. Cascieri, S. Sadowski, E. Ber, G. G. Chicchi, M. Kurtz, J. Metzger,
G. Eiermann, N. N. Tsou, F. D. Tattersall, N. J. M. Rupniak, A. R. Williams, W. Rycroft, R. Hargreaves and D. E. Maclntyre, J. Med. Chem., 1998, 41, 4607.

28 (a) A. Baeyer and V. Drewson, Ber. Dtsch. Chem. Ges., 1882, 15, 2856; (b) J. Kamlet, Ind. Eng. Chem., 1944, 16, 362; (c) Comprehensive organic name reactions and reagents, ed. $\mathrm{Z}$. Wang, Wiley: A John Wiley and sons, inc., 2009, vol. 1, pp. 136-139.

29 (a) D. J. Knobloch, E. Lobkovsky and P. J. Chirik, J. Am. Chem. Soc., 2010, 132, 15340; (b) A. F. Khattab and T. Kappe, J. Chem. Res., 2006, 9, 609; (c) J. Protasiewicz and G. D. Mendenhall, J. Org. Chem., 1985, 50, 3220; (d) Z. Du, W. B. Li, X. H. Zhu, F. Xu and Q. Shen, J. Org. Chem., 2008, 73, 8966; (e) R. Neidlein and W. Lehr, Heterocycles, 1981, 16, 1179; $(f)$ M. L. Testa, E. Zaballos and R. J. Zaragoza, Tetrahedron, 2012, 68, 9583; $(g)$ W. Ziemkowska, E. Jaskowska, E. Zygadlo-Monikowska and M. K. Cyranski, J. Organomet. Chem., 2011, 696, 2079.

30 (a) N. Hadei, E. A. B. Kantchev, C. J. O'Brien and M. G. Organ, J. Org. Chem., 2005, 70, 8503; (b) D. P. Allen, M. M. V. Wingerden and R. H. Grubbs, Org. Lett., 2009, 11, 1261; (c) M. Kuriyama, R. Shimazawa and R. Shirai, Tetrahedron, 2007, 63, 9393; (d) S. Miljanic, L. Frkanec, Z. Meic and M. Zinic, Eur. J. Org. Chem., 2006, 1323; (e) A. W. Waltman and R. H. Grubbs, Organometallics, 2004, 23, 3105; $(f)$ Y. A. Ibrahim, N. A. Al-Awadi, T. F. Al-Azemi and E. John, RSC Adv., 2014, 4, 38869.

31 (a) N. Kambe, T. Inoue, T. Takeda, S. Fujiwara and N. Sonoda, J. Am. Chem. Soc., 2006, 128, 12650; (b) T. Mizuno, M. Matsumoto and I. N. T. Hirashima, Synth. Commun., 2006, 23, 2139; (c) R. Richter, F. A. Stuber and B. Tucker, J. Org. Chem., 1984, 49, 3675.

32 A. R. Katritzky, D. P. M. Pleynet and J. R. Levell, Synthesis, 1998, 153.

33 L. E. Kaim, L. Gaultier, L. Grimaud and E. Vieu, Tetrahedron Lett., 2004, 45, 8047.

34 Z. Zhan, X. Cheng, X. Ma, J. Li, L. Hai and Y. Wu, Tetrahedron, 2015, 71, 6928.

35 (a) K. Stott, J. Stonehouse, J. Keeler, T.-L. Hwang and A. J. Shaka, J. Am. Chem. Soc., 1995, 117, 4199; (b) K. Stott, J. Keeler, Q. N. Van and A. J. Shaka, J. Magn. Reson., 1997, 125, 302.

36 T. Bally and P. R. Rable, J. Org. Chem., 2011, 76, 4818.

37 M. J. Frisch, G. W. Trucks, H. B. Schlegel, G. E. Scuseria, M. A. Robb, J. R. Cheeseman, V. G. Zakrzewski, J. A. Montgomery Jr, R. E. Stratmann, J. C. Burant, et al., Gaussian 03, Revision A.6, Gaussian, Inc, Pittsburgh, PA, 2003. 